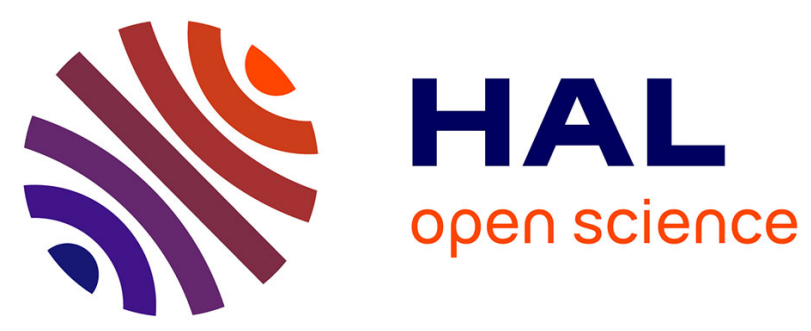

\title{
A clone of the emergent Streptococcus pyogenes emm89 clade responsible for a large outbreak in a post-surgery oncology unit in France
}

Céline Plainvert, Magalie Longo, Elise Seringe, Benjamin Saintpierre, Elisabeth Sauvage, Laurence Ma, Johann Beghain, Nicolas Dmytruk, Gislène Collobert, Eric Hernandez, et al.

\section{To cite this version:}

Céline Plainvert, Magalie Longo, Elise Seringe, Benjamin Saintpierre, Elisabeth Sauvage, et al.. A clone of the emergent Streptococcus pyogenes emm89 clade responsible for a large outbreak in a postsurgery oncology unit in France. Medical Microbiology and Immunology, 2018, 207 (5-6), pp.287-296. 10.1007/s00430-018-0546-1 . inserm-01955462

\section{HAL Id: inserm-01955462 https://www.hal.inserm.fr/inserm-01955462}

Submitted on 14 Dec 2018

HAL is a multi-disciplinary open access archive for the deposit and dissemination of scientific research documents, whether they are published or not. The documents may come from teaching and research institutions in France or abroad, or from public or private research centers.
L'archive ouverte pluridisciplinaire HAL, est destinée au dépôt et à la diffusion de documents scientifiques de niveau recherche, publiés ou non, émanant des établissements d'enseignement et de recherche français ou étrangers, des laboratoires publics ou privés. 
REVISED MMIM-D-18-00037

2

3 A clone of the emergent Streptococcus pyogenes emm89 clade responsible for a large

4 outbreak in a post-surgery oncology unit in France

6 Céline Plainvert ${ }^{1,2,3,4}$, Magalie Longo ${ }^{1,2,3}$, Elise Seringe ${ }^{5}$, Benjamin Saintpierre ${ }^{1,2,3}$, Elisabeth

7 Sauvage ${ }^{6,7}$, Laurence $\mathrm{Ma}^{8}$, Johann Beghain ${ }^{9}$, Nicolas Dmytruk ${ }^{4}$, Gislène Collobert ${ }^{4}$, Eric

8 Hernandez $^{10}$, Pascal Astagneau ${ }^{5,11}$, Philippe Glaser ${ }^{6,7}$, Frédéric Ariey ${ }^{1,2,3}$, Claire Poyart ${ }^{1,2,3,4}$

9 and Agnès Fouet ${ }^{1,2,3}$

${ }^{1}$ INSERM, U1016, Institut Cochin, Paris, 75014, France

${ }^{2}$ Université Sorbonne Paris Descartes, Paris, 75014, France

${ }^{3}$ CNRS (UMR 8104), Paris, 75014 France

${ }^{4}$ Assistance Publique Hôpitaux de Paris, Service de Bactériologie, Centre National de Référence des Streptocoques, Groupe Hospitalier Paris Centre Cochin-Hôtel Dieu-Broca, Paris, 75014 France.

${ }^{5}$ Assistance Publique Hôpitaux de Paris, Centre de Prévention des infections associées aux soins, F75014 Paris, France

${ }^{6}$ Institut Pasteur, Unité Ecologie et Evolution de la Résistance aux Antibiotiques, Paris, 75724 France

${ }^{7}$ CNRS UMR3525, Paris, 75724 France

$10{ }^{8}$ Institut Pasteur, Pôle Biomics, Paris, 75724 France

${ }^{9}$ Institut Pasteur, GGIV unit Paris 75724 France

${ }^{10}$ Centre Médical de Forcilles, 77150 Lesigny, France

${ }^{11}$ : Sorbonne université, Faculté de médecine, APHP, Pitie-Salpétrière, F75013 Paris, France 
11 Correspondence: Claire Poyart, Telephone, 331584115 60; Fax, 33154411548

12 claire.poyart@aphp.fr

13 Keywords (3 - 10): Group A Streptococcus; emm89; emerging clade; phylogeny; biofilm;

14 bacterium-cell interaction; outbreak

15

16 Running title $(<50)$ : emm89 GAS outbreak

17 


\section{Abstract}

19 An outbreak of nosocomial infections due to Streptococcus pyogenes (Group A 20 Streptococcus; GAS) occurred in a post-surgery oncology unit concerned more than 60 21 patients and lasted 20 months despite enhanced infection control and prophylaxis measures. 22 All GAS strains were characterized (emm genotype, toxin gene profile and pulse-field gel 23 electrophoresis subtype). Selected strains were sequenced and phylogenetic relationship 24 established. Capacity to form biofilm and interaction with human pulmonary epithelial cells and macrophages were determined. Twenty-six GAS strains responsible for invasive infections (II) and 57 for non-II or colonization were isolated from patients $(n=66)$ or healthcare workers $(n=13)$. Seventy strains shared the same molecular markers and 69 the same PFGE pattern; 56 were sequenced. They all belonged to the emerging emm89 clade 3; all but one were clonal. Whole genome sequencing identified 43 genetic profiles with sporadic mutations in regulatory genes and acquired mutations in two structural genes. Except for two regulatory gene mutants, all strains tested had the same biofilm formation capacity and displayed similar adherence and invasion of pulmonary epithelial cells and phagocytosis and survival in human macrophages. This large outbreak of GAS infection in a post-surgery oncology unit, a setting that contains highly susceptible patients, arose from a strain of the emergent emm89 clade. No relationship between punctual or acquired mutations, invasive status and strain phenotypic characteristics was found. Noteworthy, the phenotypic characteristics of this clone account for its emergence and its remarkable capacity to elicit outbreaks. 
INTRODUCTION

41 Group A Streptococcus (GAS, Streptococcus pyogenes) is a human pathogen causing a broad range of diseases, non-invasive and invasive diseases as well as autoimmune sequelae, with an estimated 517,000 deaths per year [1]. These can be community acquired or be the consequence of outbreaks in human settings with high proximity such as schools, military barracks or hospital wards. Residents of the latter are at increased risk of acquiring GAS infections because of underlying medical conditions favorable to streptococcal infections [2]. GAS strains are extremely diverse and currently the most widely used typing-method for GAS strains relies on the 5 'end of the $\mathrm{emm}$ gene sequence that encodes the hypervariable amino-terminus region of the M protein, a surface exposed virulence factor [3]. Among more than $200 \mathrm{emm}$-types defined, the most frequently associated with invasive infections in

51 Europe are emml, emm28 and emm89, with variable distribution worldwide [4, 5]. Epidemiological studies have evidenced the emergence of given genotypes and more precisely of clones as leading cause of disease in Europe or the United States. Such was the case with the emm1 type and the M1T1 clone in the late 1980s ([6] and references herein) and more recently of the emm89 type exemplified by the emergent clade or clade 3 variant $[7,8]$. The emergence of emm89 strains, most probably of the same clone, has been described in multiple geographic locations [9-16]. Although emm89 strain recovery from outbreaks has been described previously [17-19], the newly emerging emm89 clone has not been decisively implicated in major outbreaks. In 2012 in a follow-up care and rehabilitation post-surgery oncology unit in France, GAS infections were recorded at an unusual level and, although state-of-the-art prophylaxis measures were taken, this outbreak lasted twenty months. We investigated at the molecular

63 level the cause of this outbreak by analyzing all isolates, be they from invasive or non64 invasive diseases or colonization. The strains were genetically characterized and, using 
65 biological and date criteria, fifty-six strains were chosen and sequenced. To shed light on 66 possible reasons for this long-lasting outbreak we investigated whether the GAS strains 67 involved had specific properties in terms of biofilm formation capacity, interactions with 68 human lung epithelial cells or human macrophages.

69 
MATERIALS AND METHODS

\section{Case definition}

Invasive disease is defined through the isolation of GAS from normally sterile sites or the isolation of GAS from non-sterile sites (e.g., sputum, wounds) in the presence of either

74 streptococcal toxic shock syndrome or necrotizing fasciitis and non-invasive disease through (http://www.cdc.gov/ncdidod/biotech/strep/doc.htm)

[3]. All strains were tested by a multiplex PCR method for the presence of genes encoding the toxins or superantigens SpeA, 


\section{Bacterial growth conditions}

96 GAS isolates were grown at $37^{\circ} \mathrm{C}$ without agitation in Todd-Hewitt broth supplemented with $0.5 \%$ Yeast extract (THY) or in Brain Heart Infusion (BHI) (Difco). Bacteria were collected in mid-log phase, washed once with sterile phosphate-buffered saline (PBS), and diluted to the required inoculum; the number of viable bacteria was determined by counting the colony forming units (CFUs) after plating dilutions on THY agar.

\section{GAS strain sequencing}

103 Chromosomal DNA was extracted using the MasterPure ${ }^{\mathrm{TM}}$ Gram-positive DNA purification 104 kit (Tebu-Bio) and sequenced using the Illumina technology, with read length of $100 \mathrm{nt}$ and a 105 more than 100 fold-coverage. Libraries were constructed by using the Illumina TrueSeq kit 106 following the manufacturer's instructions. Illumina short reads were assembled by using the Velvet software [21]. Strain 20120499 considered as the outbreak index case was used as a reference for SNPs calling. Contigs of strains 20120499 were ordered by aligning them to the complete genome sequence of strain MGAS315 (emm1, accession number NC_004070.1, no

110 emm89 complete sequence being available at the time of this analysis) using the Mauve

111 software [22]. Each strain was analyzed for SNPs, small indels and deletions compared to strain 20120499 by using the BRESEQ software (pmid 24838886), which allowed the

113 identification of errors in the Velvet assembly. Sequence specific to each strain was analyzed 114 by assembling unmapped reads with Velvet. Accession numbers pending.

\section{Reconstruction of large insertion from fastq files.}

117 A Perl software named iSeGWalker was newly developed to accomplish a de novo genome 118 reconstruction from the reads file in fastq format [23]. The search step is an exact-matching 119 reads selection using a regular expression (seed) and a simple Perl script, reading each 
120

121

122

\section{Phylogeny analysis}

125 Phylogenetic analyses were performed after alignment of all strains against the new

126 reconstructed sequence as reference. The evolutionary history was inferred using the 127 Neighbor-Joining method [24]. The evolutionary distances were computed using the 128 Maximum Composite Likelihood method [25] and are in the units of the number of base 129 substitutions per site. The rate variation among sites was modeled with a gamma distribution 130 (shape parameter $=1$ ). The differences in the composition bias among sequences were 131 considered in evolutionary comparisons [26]. All positions containing gaps and missing data 132 were removed for each sequence pair. Evolutionary analyses were conducted in MEGA7 [27].

\section{$134 \quad$ PCR amplification}

135 The DNA sequence surrounding the has operon was amplified using the oligonucleotides 136 Has-M89_F CTGTGCCACTAAGACTCTTT and Has-M89_R CGGACAGGTGCAGTTGG137 TTT using the GoTaq ${ }^{\circledR}$ Green Master Mix with an elongation time of 4 min.

\section{Biofilm formation assay}

140 Overnight cultures grown in BHI, for optimal abiotic biofilm formation as described in [28], 141 were diluted to an optical density at $600 \mathrm{~nm}\left(\mathrm{OD}_{600}\right)$ of 0.06 and $180 \mu \mathrm{L}$ were distributed in 142 quadruplet in $96-$ well plates. After $24 \mathrm{~h}$ static growth at $37^{\circ} \mathrm{C}$, one plate was used to record 143 the $\mathrm{OD}_{595}$ after resuspension while the other one was treated with crystal violet as described 144 by Köller et al. [28] except that the aqueous 1\% SDS solution was replaced by a $80 / 20$ 
145 ethanol/acetone solution. Biofilm formation capacity was defined as the ratio of the $\mathrm{OD}_{595}$

146 elicited by crystal violet and the $\mathrm{OD}_{595}$ due to growth on the control plate. The experiment 147 was carried out at least in triplicate.

149 Growth kinetics.

150 Overnight cultures grown in THY were diluted 1:100 in fresh THY and 150 $\mu \mathrm{L}$ were 151 distributed in quadruplet in 96-well plates. The plates were incubated at $37^{\circ} \mathrm{C}$ in a Thermo 152 Scientific Multiskan ${ }^{\mathrm{TM}}$ GO microplate spectrophotometer. $\mathrm{OD}_{600}$ was measured every 10 min 153 after agitation.

Human lung epithelial cell culture and adhesion and invasion assays

156 A549 cells were grown in DMEM-Glutamax 10\% Fetal Calf Serum (FCS) in 24-well plates during 2 days, washed with PBS and infected with mid-logarithmic GAS resuspended in RPMI cell-culture medium at a multiplicity of infection (MOI) of 1 bacterium / cell. For adhesion assays, the plates were centrifuged $5 \mathrm{~min}$ at $1000 \mathrm{rpm}$ and incubated $1 \mathrm{~h}$ at $4{ }^{\circ} \mathrm{C}$; the non-adherent extracellular bacteria were then eliminated removing the culture medium and washing three times with sterile PBS. For internalization assays, the plates were further incubated $45 \mathrm{~min}$ at $37^{\circ} \mathrm{C}, 5 \% \mathrm{CO}_{2}$, and the adherent extracellular bacteria were subsequently killed by incubation with fresh medium containing $50 \mathrm{U} / \mathrm{mL}$ penicillin/ and $50 \mu \mathrm{g} / \mathrm{mL}$ 164 streptomycin for 30 min. After washing with PBS A549 cells were lysed with $1 \mathrm{~mL}$ cold 165 sterile distilled water and serial dilutions were plated on THY plates and the number of CFUs was determined after $24-48$ hours growth at $37^{\circ} \mathrm{C}$. For all experiments, 4 or 5 independent assays in duplicate were carried out for each bacterial isolate. 
170 Human macrophage culture and infection assays

171 To differentiate the THP-1 into macrophages, $2.10^{5}$ to $4.10^{5}$ cells were inoculated in RPMI 172 GluMax supplemented with 10\% FCS and 200 nM PMA (Phorbol 12-myristate 13-acetate) 173 (Sigma, P1585). After 3 days at $37^{\circ} \mathrm{C}, 5 \% \mathrm{CO}_{2}$, the medium was replaced with RPMI 174 GluMax supplemented with $10 \%$ FCS and the incubation extended for 5 days. Cells were then 175 infected with mid-logarithmic GAS resuspended in RPMI medium at a MOI of 1 . The 176 phagocytosis and survival assays were carried out as previously described [29].

178 Statistical analyses.

179 Significance of differences in biofilm formation capacities were assessed by Student's t-test. 180 That of cellular adhesion, invasion, phagocytosis and survival were assessed by Repeated 181 measures Anova and Turkey's multiple comparison test, GraphPad software. 


\section{Outbreak description}

186 From $16^{\text {th }}$ April through $7^{\text {th }}$ May 2012, 4 cases of invasive GAS infections were recorded in a 187 100-bed post-surgery oncology unit (Fig.1). To limit secondary cases, epidemiological 188 investigations were started among the patients and healthcare workers to determine their 189 status against GAS. Although appropriate hygiene measures were taken this outbreak lasted 190 until $23^{\text {rd }}$ December 2013 concerned 66 cases and ended after mass chemoprophylaxis by 191 azithromycin $500 \mathrm{mg}$ per os once daily for 3 days and enhanced infection control such as 192 mask wear, hand hygiene and the implementation of appropriate disinfection measures for 193 tracheostomy cannula.

194 Almost all the patients had surgery for an oral cancer and were in second line of treatment 195 with radiotherapy. Men accounted for $83 \%(55 / 66)$ of the patients and the mean age were 61 196 year-old (range 24-87) and 67.5 year-old (52-83) for men and women, respectively (Table S1 and data not shown).

198 During the outbreak, 26 cases of GAS invasive infections were identified, 13 bacteremia, 11 199 pneumonias and 2 necrotizing fasciitis, among which 5 (19.2\%) died. During the 200 epidemiological surveillance, 57 non-invasive GAS strains were isolated; 44 from patients 201 and 13 from healthcare workers with direct patient-care responsibilities (Table S1).

202 GAS patients' strains, were isolated from blood cultures $(n=13)$, oropharynx $(n=9)$, wounds $203(n=13)$, gastrostomy sites $(n=8)$, tracheostomy cannula $(n=8)$, respiratory specimen $(n=18)$, 204 and lymphadenectomy $(\mathrm{n}=1)$. All thirteen GAS-positive cultures from healthcare workers 205 were from the oropharynx. A total of 83 GAS strains were thus isolated between April 2012 206 and December 2013 including 26 invasive, 38 non-invasive, and 19 from colonization. An 207 invasive respiratory device was present in 13/26 (50\%) patients with an invasive infection and 208 in only $12 / 44(27 \%)$ patients colonized or without an invasive infection, $p=0.049$. 
210 All strains were genotyped (Table S1). All invasive but one (25/26) and 51 out of 57 non-

211 invasive or colonization strains shared the same emm89 genotype. Sixty-nine/76 (91\%) 212 emm89 GAS strains, be they invasive, non-invasive or colonization strains, were 213 indistinguishable by PFGE displaying an 89-A4 pattern according to our nomenclature (Table 214 S1 and data not shown). Patterns of the seven emm89 remaining strains showed a maximum 215 of four differences. All 76 emm89 strains displayed a low-level resistance to aminoglycosides 216 (kanamycin and gentamicin) and were susceptible to $\beta$-lactams, vancomycin, macrolides and 217 tetracycline except two non-89-A4 strains that were resistant to tetracycline due to the 218 presence of $\operatorname{tet}(\mathrm{M})$ determinant located on $\mathrm{Tn} 916$ related conjugative transposon.

219 Thus, whereas non-89-A4 PFGE and non-emm89 strains were occasionally isolated, 69/83

220 GAS strains isolated throughout the considered period shared the same emm89 genotype 89221 A4 PFGE pattern. This argued for an outbreak origin of these strains; the other strains were 222 considered outside the outbreak. To further analyze these emm89 89-A4 strains, a total of 56 223 were randomly chosen for sequencing among a sub-collection selected according to date and 224 clinical criteria as follows: a balanced distribution, 22/26 and 34/50 isolated in 2012 and 2013, 225 respectively, including the first, index case in 2012, and last of each year, and 9 and 11 226 invasive infections from 2012 and 2013, respectively. For sake of comparison, we added an 227 unrelated emm89 invasive strain isolated in 2012 (20120456) as well as three clinical isolates 228 isolated at the same post-surgery oncology unit but in 2014 (20141462, 20141463 and 229 20140469; Table S2).

231 Sequence analysis and phylogeny of the GAS outbreak isolates

232 The alignment of three fastq files, including that of the index case strain (20120499), to H293 233 emm89 genomic sequence (NC_002737.2), and the use of the Tablet software [30], led to the 
234 identification of 5 areas of possible chromosomal rearrangement sites based on a relative

235 increase in the diversity of these loci. We then reconstructed the new reference genome (see 236 Materials and Methods) (1 747907 base pairs) and realigned all the fastq files on it. The 237 genomes are $99 \%$ identical on a 99\% coverage to that of MGAS27061 (accession number 238 NZ_CP013840.1), indicating that these emm89 clinical isolates belong to the new emm89 239 clade 3, lacking the has operon and harboring the same mutation in the slo-nga promoter 240 region $[13,31]$. To get insight into the emergence of this new clade in France we sought, in a 241 collection of emm89 French strains, when the has operon loss could have occurred. The DNA 242 fragment encompassing the has operon was amplified from 15 unrelated strains received by 243 the CNR-Strep during the last 15 years (data not shown). In three of these strains, including 244 one from 2003 the has operon was absent, suggesting the presence of the new clade in France 245 already at that time.

246 For phylogenetic analyses we used the whole genome alignment of 61 strains: we combined 247 genomic data obtained from 56 isolates of the 2012-2013 outbreak, one emm89 control strain 248 independent of this outbreak (20120256), three strains isolated in 2014 at a time-related 249 distance from the outbreak, and the reference MGAS27061 an emm89 clade 3 strain [7, 8] 250 (Fig.2; Table S2). This tree (Fig.2) clearly shows the monophyletic character of the $2512012 / 2013$ outbreak, well separated from the isolates collected at the same site but in time 252 away from the outbreak (2014 strains). The control strain 20120456 is phylogenetically more 253 distant to the index case strain (42 SNPs compared to the 20120499 index strain) than all 254 other strains. Furthermore, the fact that the reference strain MGAS26761 is positioned 255 between the branches 2012/2013 and 2014, reinforces the independence of the two events and 256 strongly suggests the extinction of the outbreak episode of 2012/2013 (Fig. 2).

257 The tree covering the 55 outbreak strains (Fig.3) presents three separate subclones linked to 258 their date of isolation. Of the three main subclones, two are composed solely of 2012 strains 
259 (subclones 1 and 2 2012). Among strains belonging to subclone 3 2013, two, 20121368 and

26020121198 , were the last isolated in 2012, in fact during the last trimester of 2012.

261 Also they have lost the phage harboring speC, however loss of a phage being compatible with

262 a convergence event. The strain 20130948 appears to have a quite peculiar evolutionary

263 history and lies apart from all subclones. Despite these exceptions this topography is highly

264 compatible with an epidemic signature: most strains deriving from a unique colonization

265 event occurring in 2012. The emm89 outbreak subclone was termed emm89-F.

266 Forty-three genetic profiles were defined (Table S3). At the gene level, four mutations

267 stabilized over the outbreak period; two occurred in intergenic regions and the two others in

268 genes involved in bacterial metabolism, the oxaloacetate decarboxylase beta chain (ODC),

269 found in 36 strains from $8^{\text {th }}$ September 2012 onward, and the shikimate 5-dehydrogenase I 270 alpha (S5D), found in 33 strains from $25^{\text {th }}$ January 2013 onward. Ten non-stabilized

271 mutations were also found in the gene encoding the serum opacity factor (SOF) among 29

272 strains. Six non-fixed mutations were also found among 8 strains in the gene encoding the 273 CovS regulatory protein. No mutation or group of mutations could be related to the strain 274 invasive status.

\section{Phenotypic characterization of the emm89-F strains}

277 Isolates of the emm89-F clone were characterized for phenotypes relevant to the clinical 278 settings of this outbreak, i. e. growth capacities, abiotic biofilm formation, interactions with 279 lung epithelial cells and macrophages. Strains were chosen to cover all the branches of the 280 genetic tree and to, altogether, harbor all mutation profiles (Fig. 3, Table S1). 
285 As mentioned a significant correlation between the occurrence of invasive GAS infections and the presence of a medical respiratory device was observed among infected patients. (Table S1). The recognition that biofilm formation by GAS is involved in persistence (for review [32]) and that biofilm formation varies among strains within emm genotypes [33] prompted us to assay the capacity of these clinical isolates to form biofilm on abiotic surface (Fig. 4). All strains were able to form biofilms and most of them in similar amounts; no difference was observed between strains sampled from tracheostomy cannula and from other sites. The stronger biofilm producers are mutated in either the global positive regulator gene $m g a$, yielding values slightly above those of the other strains, or, and more impressively, in $\operatorname{cov} S$, the sensor of the CovRS two component system known to negatively regulate the expression of multiple virulence genes [34].

We also assessed whether GAS biofilm formation capacities increased during the outbreak.

297 To that aim we calculated a trend line, after removing the strains mutated in regulatory genes 298 (Fig. S1). It slightly raises $(y=0.0362 x+8.8)$ but displays no great change in biofilm 299 formation throughout the outbreak.

300 To assess whether the outbreak isolates were weak or robust biofilm formers, their capacity to 301 form biofilms was compared to that of other unrelated clinical isolates (Fig. 4, Table S2). 302 Biofilm formation being dependent on the CovRS regulatory system, we included only covR $303 \operatorname{covS}$ wild-type isolates from other studies and calculated the mean value for the outbreak 304 isolates excluding all regulatory mutants [35]. The clinical isolates collected during the 305 outbreak formed substantial more biofilms compared to isolates from other emm types, $(p=$ 306 0.001). In contrast, no difference was observed when comparison was established with other 307 emm89 isolates, also belonging to the emergent clade $3[7,8]$, (data not shown) indicating that 308 this may be a specific trait to the emerging emm89 clones. 
In conclusion these results show that emm89 outbreak isolates are more robust biofilm

310 formers than non-emm89 isolates; this property could account and contribute for the current 311 emergence and spread of this clone.

\section{Adhesion and invasion into lung epithelial cells}

314 The pathologies observed during the outbreak such as respiratory infections prompted us to analyze the adhesion and invasion of selected clinical isolates into human A549 lung epithelial cells. Four strains were selected, in addition to the index case (IC; 120499), using different criteria to ensure a representative sampling: dispersion throughout the genetic tree and the presence of different mutations, SOF (120811), ODC (120747), S5D (130922) and the regulator CovS (120619) (Fig. 3 and Fig. S1 and Table S1). We first assessed that these strains displayed no growth difference in laboratory conditions (data not shown).

Adhesion capacities of these strains were determined and compared to that of an unrelated emm89 strain previously studied [29]. No significant difference in the adhesion capacity of

323 these strains was observed (Table 1). In contrast, a difference was noted when invasion was 324 compared: the invasion of the $\operatorname{cov} S$ mutant isolate was lower than that of all the other strains 325 and was significantly lower than that of the emm89 control strain (Table 1). This suggests that 326 these clinical isolates possessed no specific properties in their interaction with pulmonary 327 cells compared to other unrelated GAS emm89 clinical isolates and did not acquire any, 328 through their mutations detected.

\section{Phagocytosis and survival within macrophages}

331 Macrophages are critical host defense cells. We have previously shown that emm89 isolates 332 were more phagocytozed by murine macrophages than non-emm 89 strains, that they were 333 differentially phagocytozed depending on their invasive status and that there was a variability 
334 within the invasive and non-invasive strains [29]. Consequently, we analyzed the behavior of

335 the outbreak clinical isolates towards uptake and survival in the physiologically relevant 336 THP-1 human macrophages and compared it to that of the emm89 control strain (Fig. 5).

337 Except for the $\operatorname{cov} S$ mutant strain, which seemed less phagocytozed than all other strains and 338 was significantly less phagocytozed than the SOF and S5D strains, all outbreak isolates and 339 the control strain were similarly phagocytozed by THP1 macrophages (Fig. 5A).

340 We then tested the survival of these isolates in THP-1 (Fig. 5B). Only the covS mutant strain 341 survived, at T2, less than other strains, and significantly less than the index isolate.

342 This indicates that the outbreak strains and the emm89 control isolate behaved similarly 343 towards the host defense elicited by the human macrophages. 


\section{DISCUSSION}

346 This is, to our knowledge, one of the largest outbreaks of GAS infection in a single health

347 care facility among highly vulnerable patients with oral cancer. Here we describe a

348 remarkably long outbreak linked to the persistence and spread of a clone in both patients and

349 healthcare workers. Almost all isolates from patients with GAS infection and those from

350 healthcare personnel were subtype emm89, suggesting that one GAS strain was circulating

351 throughout the healthcare facility. However, we could not determine whether a patient or an

352 infected colonized healthcare worker initiated the outbreak since investigations began three

353 weeks after notification to the authorities of the four cases of invasive infections. In France,

354 emm89 is now among the top three leading emm types, accounting for $19 \%$ of all invasive

355 infections in 2017 and the first one (23\%) for GAS clustered cases since 2011 (https://cnr-

356 strep.fr/index.php/bilans-dactivite/rapports-dactivite).

357 Using whole-genome sequencing we demonstrated that this clone belongs to the emerging

emm89 clade $3[7,8,14]$. Interestingly, most but not all isolates could be phylogenetically

359 grouped on the basis of their sampling year (Fig. 2). The exceptions suggest that some clones

360 reappeared after a few months during which they were asymptomatically carried. Several data

361 report that this emm89 emerged in many countries in a closely similar time frame during the

362 first decade of the 2000s, accompanied by a swift from clade 1 to clade 2 and subsequent

363 emergence of clade 3 [12].

364 The extent of this outbreak and the case-fatality rate of $19 \%$ among patients with invasive 365 disease prompted us to shed light on the advantages brought by this specific clone; we studied

366 phenotypic traits such as biofilm formation and interaction with human pulmonary cells or 367 macrophages. The similar high level of biofilm formation displayed by the outbreak and other 368 emm89 clones compared to other emm-type strains could be a consequence of all strains 369 belonging to the emerging emm89 clade. Indeed, emm89 strains were not described as high 
biofilm producer by Koller et al. [28], and this could be linked to their strain panel, sampled

371 between 2001 and 2006, that is before the full emergence of this clone [7, 28]. Here we show 372 that the emm89-F clone, which is uncapsulated, is a robust biofilm former confirming that 373 capsule is dispensable for abiotic biofilm formation [35, 36]. The emm89-F mga mutants, 374 Mga being a global transcriptional activator including of the $\mathrm{M}$ and $\mathrm{M}$-like encoding genes, 375 yielded slightly higher biofilm production than the wild-type emm89-F isolates. M and M-like 376 proteins most often contribute to biofilm formation, which is nevertheless emm-type 377 dependent [35-37]; a weaker M and M-like protein production in an emm89 background may 378 have, as in the emm2 background, little influence on biofilm formation [37]. Furthermore, a 379 variation in the abundance of Mga-controlled products, other than the $\mathrm{M}$ and $\mathrm{M}$-like proteins, 380 could account for the biofilm increase. CovRS is a two-component system that controls, mainly repressing, the expression of $15 \%$ of the GAS genome. Several covS-mutant strains produce less, and others more biofilm than their wild-type counterparts, suggesting that the consequences of mutations in $\operatorname{cov} S$ on biofilm formation are strain-dependent $[35,38]$. The emm89-F covS mutant isolates were particularly robust biofilm formers supporting the strain385 dependent contribution of CovS to biofilm formation and demonstrating that CovRS386 controlled products other than the capsule are involved in biofilm formation.

387 Several reports suggested that acapsular emm89 clade-associated GAS strains may have 388 acquired potential for long-term colonization [7, 13]. Moreover, the increased expression of 389 SLO and NADase by this clade could enhance internalization and intracellular bacterial 390 survival in epithelial cells, theoretically providing protection from natural antimicrobial 391 peptides and antibiotic treatment. Adhesion and invasion experiments on human epithelial 392 lung cells performed on a representative sampling did not reveal major difference among 393 strains except for the $\operatorname{cov} S$ strain for which, if adhesion was similar to other strains, its 394 capacity to invade cells was affected. Furthermore, there was a tendency for $\operatorname{cov} S$ strain to be 
395 less phagocytozed and to survive less than the others in the macrophage. Altogether this

396 meant a disadvantage for this strain for which epithelial cells and macrophages would not act

397 as a reservoir and promote a carrier state. This result is consistent with the report that covS

398 strains are badly transmitted [39], thus accounting for sporadic apparition of covS mutations

399 during this outbreak.

400 In this outbreak the majority of the patients had undergone surgery associated with

401 radiotherapy for oral cancer and belongs to a disadvantaged and highly vulnerable population.

402 These features, could explain the very high rate of transmission in fragile patients where

403 promiscuity was highly problematic.

404 In conclusion, this report adds France to the growing list of European countries and North

405 America where, by whole genome sequencing, emm89 clade 3 strains have been demonstrated

406 to an emergent clone eliciting GAS infections. It strengthens the fact that the spreading of this

407 clone is worldwide and emphasizes the importance of epidemiological monitoring by

408 reference centers and health authorities. It confirms the relevance, power and robustness of

409 the whole genome sequencing approach for epidemiological analysis of major outbreaks and

410 its routine use for surveillance. 


\section{Conflict of interest statement}

413 The other authors declare no competing financial interests.

414 Acknowledgements

415 This work was supported by Santé Publique France, INSERM, CNRS, Université Paris

416 Descartes and by the High Council for Scientific and Technological Cooperation between

417 France-Israel "Complexity in Biology" program.

418 Authorship/Contributor

419 Conceived and designed the study: Céline Plainvert, Agnès Fouet and Claire Poyart. Provided 420 clinical and epidemiological data Elise Seringe, Eric Hernandez and Pascal Astagneau. 421 Performed the bacteriological analyses Magalie Longo, Nicolas Dmytruk and Gislaine 422 Collobert. Performed biofilms and phagocytosis analyses Magalie Longo. Performed Whole 423 genome sequencing of bacterial strains Benjamin Saintpierre, Elisabeth Sauvage, Laurence 424 Ma and Johann Beghain. Analysed genomes data Philippe Glaser, Frédéric Ariey and Agnès 425 Fouet. Wrote the manuscript Claire Poyart, Céline Plainvert and Agnès Fouet. 


\section{REFERENCES}

4301 Carapetis JR, Steer AC, Mulholland EK, Weber M. The global burden of group A streptococcal diseases. Lancet Infect Dis. 2005; 5: 685-694.

4322 Deutscher M, Schillie S, Gould C, et al. Investigation of a group A streptococcal outbreak among residents of a long-term acute care hospital. Clin Infect Dis. 2011; 52: 988-994.

3 Beall B, Facklam R, Thompson T. Sequencing emm-specific pcr products for routine and accurate typing of group A streptococci. J Clin Microbiol. 1996; 34: 953-958. Plainvert C, Doloy A, Loubinoux J, et al. Invasive group a streptococcal infections in adults, france (2006-2010). Clin Microbiol Infect. 2012: 702-710.

Steer AC, Law I, Matatolu L, Beall BW, Carapetis JR. Global emm type distribution of group A streptococci: Systematic review and implications for vaccine development. Lancet Infect Dis. 2009; 9: 611-616.

4426 Nasser W, Beres SB, Olsen RJ, et al. Evolutionary pathway to increased virulence and epidemic group a Streptococcus disease derived from 3,615 genome sequences. Proc Natl Acad Sci U S A. 2014; 111: E1768-1776.

Turner CE, Abbott J, Lamagni T, et al. Emergence of a new highly successful acapsular group a Streptococcus clade of genotype emm89 in the united kingdom. MBio. 2015; 6: e00622.

Zhu L, Olsen RJ, Nasser W, de la Riva Morales I, Musser JM. Trading capsule for increased cytotoxin production: Contribution to virulence of a newly emerged clade of emm89 Streptococcus pyogenes. MBio. 2015; 6: e01378-01315. the same successful clade among distinct populations of emm89 Streptococcus pyogenes in multiple geographic regions. MBio. 2015; 6: e 01780-01715.

10 Musser JM, Zhu L, Olsen RJ, Nasser W. Musser et al. Reply to "emergence of the same successful clade among distinct populations of emm89 streptococcus pyogenes in multiple geographic regions". MBio. 2015; 6: e01838-01815.

1 Turner CE, Lamagni T, Holden MT, et al. Turner et al. Reply to "emergence of the same successful clade among distinct populations of emm89 Streptococcus pyogenes in multiple geographic regions". MBio. 2015; 6: e01883-01815. 
46012 Latronico F, Nasser W, Puhakainen K, et al. Genomic characteristics behind the 461 spread of bacteremic group a Streptococcus type emm89 in finland, 2004-2014. J Infect Dis. 2016; 214: 1987-1995.

46313 Beres SB, Kachroo P, Nasser W, et al. Transcriptome remodeling contributes to 464 epidemic disease caused by the human pathogen Streptococcus pyogenes. MBio. 2016; 7.

46614 Beres SB, Olsen RJ, Ojeda Saavedra M, et al. Genome sequence analysis of emm89 467 streptococcus pyogenes strains causing infections in scotland, 2010-2016. J Med Microbiol. 2017.

15 Chochua S, Metcalf BJ, Li Z, et al. Population and whole genome sequence based 471 characterization of invasive group a streptococci recovered in the united states during

472 2015. MBio. 2017; 8 .

473

Teatero S, Coleman BL, Beres SB, et al. Rapid emergence of a new clone impacts the population at risk and increases the incidence of type emm89 group a Streptococcus invasive disease. Open Forum Infect Dis. 2017; 4: ofx042.

19 Yang $\mathrm{P}$, Peng X, Yang J, Dong X, Zhang M, Wang Q. A probable food-borne outbreak of pharyngitis after a massive rainstorm in beijing, caused by emm89 group a streptococcus rarely found in china. Int J Infect Dis. 2013; 17: e471.

20 Tenover FC, Arbeit RD, Goering RV, et al. Interpreting chromosomal DNA restriction 485 patterns produced by pulsed-field gel electrophoresis: Criteria for bacterial strain typing. J Clin Microbiol. 1995; 33: 2233-2239.

21 Zerbino DR, Birney E. Velvet: Algorithms for de novo short read assembly using de bruijn graphs. Genome Res. 2008; 18: 821-829.

48822 Darling AC, Mau B, Blattner FR, Perna NT. Mauve: Multiple alignment of conserved genomic sequence with rearrangements. Genome Res. 2004; 14: 1394-1403. 2016; 113: 9834-9839. 
49324 Saitou N, Nei M. The neighbor-joining method: A new method for reconstructing $494 \quad$ phylogenetic trees. Mol Biol Evol. 1987; 4: 406-425.

49525 Tamura K, Nei M, Kumar S. Prospects for inferring very large phylogenies by using the neighbor-joining method. Proc Natl Acad Sci U S A. 2004; 101: 11030-11035.

49726 Tamura K, Kumar S. Evolutionary distance estimation under heterogeneous 498 substitution pattern among lineages. Mol Biol Evol. 2002; 19: 1727-1736.

49927 Kumar S, Stecher G, Tamura K. Mega7: Molecular evolutionary genetics analysis $500 \quad$ version 7.0 for bigger datasets. Mol Biol Evol. 2016; 33: 1870-1874.

50128 Koller T, Manetti AG, Kreikemeyer B, et al. Typing of the pilus-protein-encoding fct 502 region and biofilm formation as novel parameters in epidemiological investigations of Streptococcus pyogenes isolates from various infection sites. J Med Microbiol. 2010; 59: 442-452.

50529 Dinis M, Plainvert C, Kovarik P, Longo M, Fouet A, Poyart C. The innate immune 506 response elicited by group a Streptococcus is highly variable among clinical isolates and correlates with the emm type. PLoS One. 2014; 9: e101464.

509

30 Milne I, Stephen G, Bayer M, et al. Using tablet for visual exploration of second-

51031 Watanabe S, Sasahara T, Arai N, et al. Complete genome sequence of Streptococcus 511 pyogenes strain jmub1235 isolated from an acute phlegmonous gastritis patient. $512 \quad$ Genome Announc. 2016; 4.

51332 Young C, Holder RC, Dubois L, Reid SD. Streptococcus pyogenes biofilm. $514 \quad 2016 / 02 / 12$ edn 2016.

51533 Lembke C, Podbielski A, Hidalgo-Grass C, Jonas L, Hanski E, Kreikemeyer B. 516 Characterization of biofilm formation by clinically relevant serotypes of group a $517 \quad$ streptococci. Appl Environ Microbiol. 2006; 72: 2864-2875.

51834 Churchward G. The two faces of janus: Virulence gene regulation by covr/s in group a $519 \quad$ streptococci. Mol Microbiol. 2007; 64: 34-41.

52035 Cho KH, Caparon MG. Patterns of virulence gene expression differ between biofilm 521 and tissue communities of streptococcus pyogenes. Mol Microbiol. 2005; 57: 15455221556.

52336 Fiedler T, Koller T, Kreikemeyer B. Streptococcus pyogenes biofilms-formation, 524 biology, and clinical relevance. Front Cell Infect Microbiol. 2015; 5: 15. 
52537 Courtney HS, Ofek I, Penfound T, et al. Relationship between expression of the 526 family of $\mathrm{m}$ proteins and lipoteichoic acid to hydrophobicity and biofilm formation in $527 \quad$ Streptococcus pyogenes. PLoS One. 2009; 4: e4166.

52838 Sugareva V, Arlt R, Fiedler T, Riani C, Podbielski A, Kreikemeyer B. Serotype- and strain- dependent contribution of the sensor kinase covs of the covrs two-component system to Streptococcus pyogenes pathogenesis. BMC Microbiol. 2010; 10.

53139 Alam FM, Turner CE, Smith K, Wiles S, Sriskandan S. Inactivation of the covr/s virulence regulator impairs infection in an improved murine model of Streptococcus pyogenes naso-pharyngeal infection. PLoS One. 2013; 8: e61655.

534

535 


\section{FIGURE LEGENDS}

538 Figure 1. History of GAS isolates. Each isolate is indicated by a symbol; patient invasive

539 strain, plain black circle; patient non-invasive or colonizing strain, open circle; healthcare

540 worker, grey circle; the symbols of sequenced strains are bordered by a red line; sampling

541 months are indicted below.

542 Figure 2. Evolutionary relationships of taxa of the sequenced isolates.

543 The optimal tree with the sum of branch length $=1.78211648$ is shown. Only bootstrap 544 greater than $50 \%$ are shown. The tree is drawn to scale, with branch lengths in the same units 545 as those of the evolutionary distances used to infer the phylogenetic tree. The analysis 546 involved 66 nucleotide sequences. All positions containing gaps and missing data were 547 eliminated including the region of the $25 \mathrm{~kb}$ insertion. There were a total of 206 positions 548 (informative SNP) in the final dataset. $20120456 \mathrm{C}$ in bold red case corresponds to the control 549 strain isolated independently of the outbreak and the strain of the index case is written in 550 black bold case.

552 Figure 3. Evolutionary relationships of emm89 outbreak isolates.

553 The optimal tree with the sum of branch length $=0.00002589$ is shown. The tree is drawn to 554 scale, with branch lengths in the same units as those of the evolutionary distances used to 555 infer the phylogenetic tree. The analysis involved 55 nucleotide sequences. All positions 556 containing gaps and missing data were eliminated including the SpeC harboring $25 \mathrm{~kb}$ 557 insertion, absent from strains 20121198 and 20121368. There were a total of 1716368 558 positions in the final dataset. Sub-clone 1 (blue) and 2 (red) for 2012 are encircled and 559 identified. 12, 13, isolates sampled in 2012 and 2013 localized in the 2013 (green) and 2012 560 clades, respectively; $\Delta$, absence of the $25 \mathrm{~kb}$ speC harboring phage; IC, index case, SOF, 561 ODC, S5D, CovS, isolates with mutations in a fibronectin binding-protein, oxaloacetate 
562 decarboxylase beta chain (EC 4.1.1.3), shikimate 5-dehydrogenase I alpha (EC 1.1.1.25), the 563 sensor CovS, respectively.

565 Figure 4. The outbreak emm89-F isolates form substantial biofilms compared to other non566 emm89 GAS strains. (A) Comparison of biofilm formation of isolates from the epidemic 567 clone and of strains from other emm-types (Table S2). For each strain, the mean value of its

568 biofilm-forming capacity is indicated. It was obtained by measuring to the ratio between the 569 crystal violet elicited $\mathrm{OD}_{595}$ and that of the bacterial growth, of three to six experiments. The 570 bar corresponds to the mean value within each group. The mutants in regulatory genes were 571 not included in the calculation of the mean values but are nevertheless represented on the 572 figure. Grey closed circles 2012 strains; black closed circles, 2013 strains; symbols for the

573 five representative strains for which other phenotypes have been analyzed are highlighted; 574 larger closed circle, IC strain (CNR120499); larger open circle, CovS strain (CNR120619); 575 closed square, ODC strain (CNR120747); closed downward pointing triangle, SOF strain 576 (CNR120811; closed diamond, S5B strain (CNR130922); also open square, the emm89 577 control strain; blue circles, covS mutants; mauve circles, mga mutant strains. (Student's t-test, $578 * * * \mathrm{p} \leq 0.001$ ). (B) A tendency curve was calculated using the means shown in Fig. $3 \mathrm{~A}, \mathrm{y}=$ $5790.0362 \mathrm{x}+8.8$ excluding the mutants in regulatory genes. The sampling year is indicated 580 below. Symbols same as in Fig. 1.

582 Figure 5. Phagocytosis (A) and survival (B) of GAS emm89-F isolates in human 583 macrophages. THP-1 cells were infected with emm89-F strains or an emm89 previously 584 characterized control strain (Table S2, [29]) as described in Material and Methods and results 585 are expressed as the percentages of (A) bacterial CFUs recovered after 30 min antibiotic 586 treatment relative to the initial inoculum and (B) the percentage of phagocytozed bacteria that 
587 survived. The results represent the mean $\pm \mathrm{SD}$ of 5 independent experiments carried out in

588 triplicate for each isolate, with significance levels indicated between a given strain and the $589 \operatorname{covS}$ mutant strain types $(* \mathrm{p} \leq 0.05 ; * * \mathrm{p} \leq 0.01)$.

590

591 Figure S1. The outbreak emm89-F isolates form substantial biofilms compared to other non592 emm89 GAS strains. A tendency curve was calculated using the means shown in Fig. 4, $\mathrm{y}=$ $5930.0362 x+8.8$ excluding the mutants in regulatory genes. The sampling year is indicated 594 below. Symbols are the same as in Fig. 1. 
Table 1. Adhesion and invasion of human epithelial lung cells (A549) by emm89-F 596 isolates

597

\begin{tabular}{lllllll}
\hline & IC & ODC & SOF & S5D & CovS & M89 \\
\hline Adhesion & $40.20+/-4.6$ & $57.48+/-17.2$ & $39.40+/-13.5$ & $75.67+/-21.9$ & $41.06+/-3.2$ & $51.12+/-13.1$ \\
Invasion & $6.34+/-3.4$ & $8.64+/-4.3$ & $8.40+/-4.4$ & $9.44+/-5.0$ & $0.38+/-0.26$ & $14.31+/-6.3^{* *}$ \\
\hline
\end{tabular}

598

599 Results are expressed as the percentages of, for the adhesion, bacterial CFUs recovered after 600 three PBS washing relative to the initial inoculum and, for the invasion, bacterial CFUs 601 recovered after $30 \mathrm{~min}$ antibiotic treatment relative to the adherent CFUs. The results 602 represent the mean $\pm \mathrm{SD}$ of 3 and 5 independent experiments, respectively, carried out in 603 triplicate for each isolate, with significance levels indicated between a given strain and the $604 \operatorname{cov} S$ mutant strain types $(* * \mathrm{p} \leq 0.01)$ 


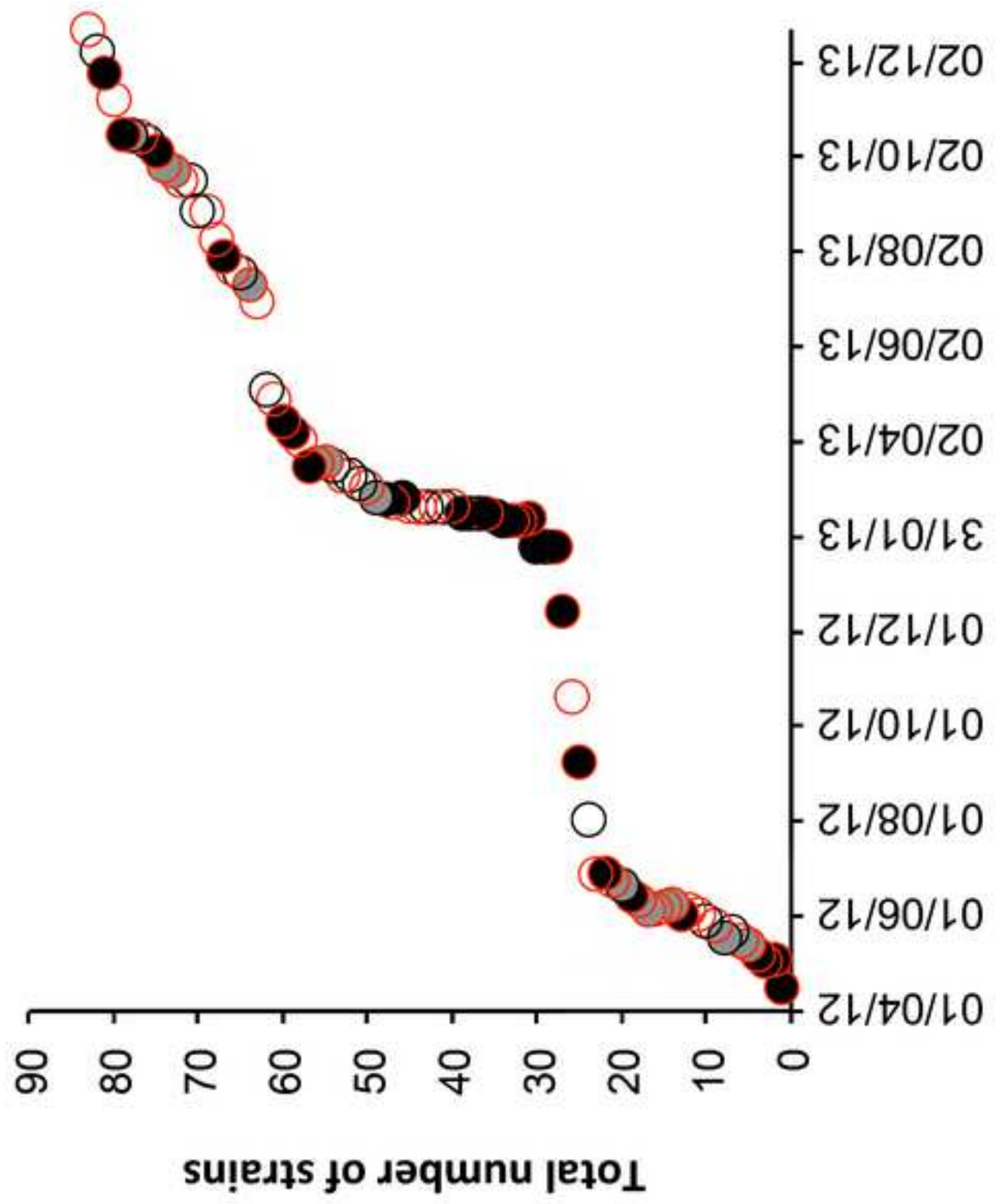




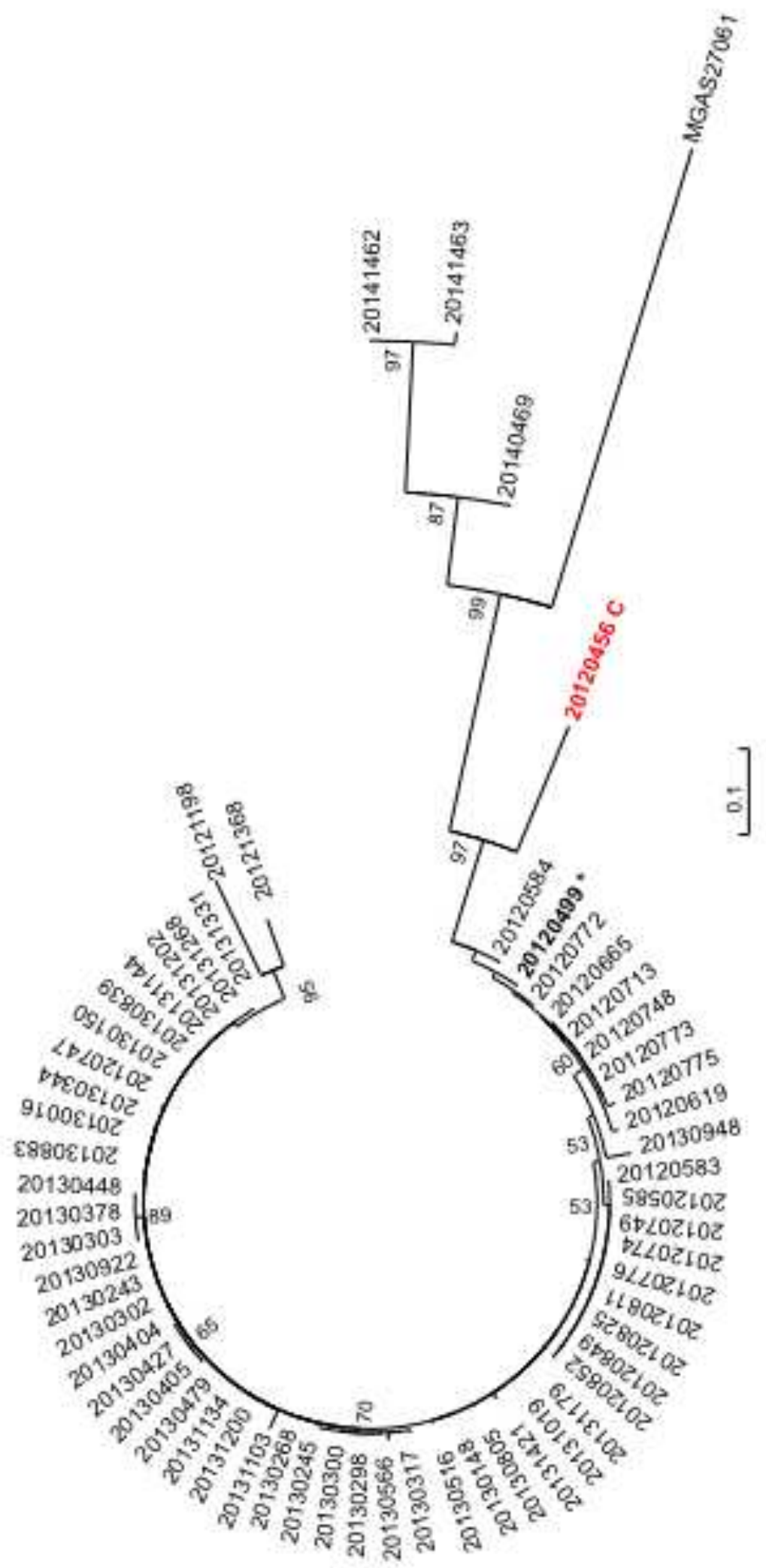

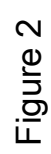




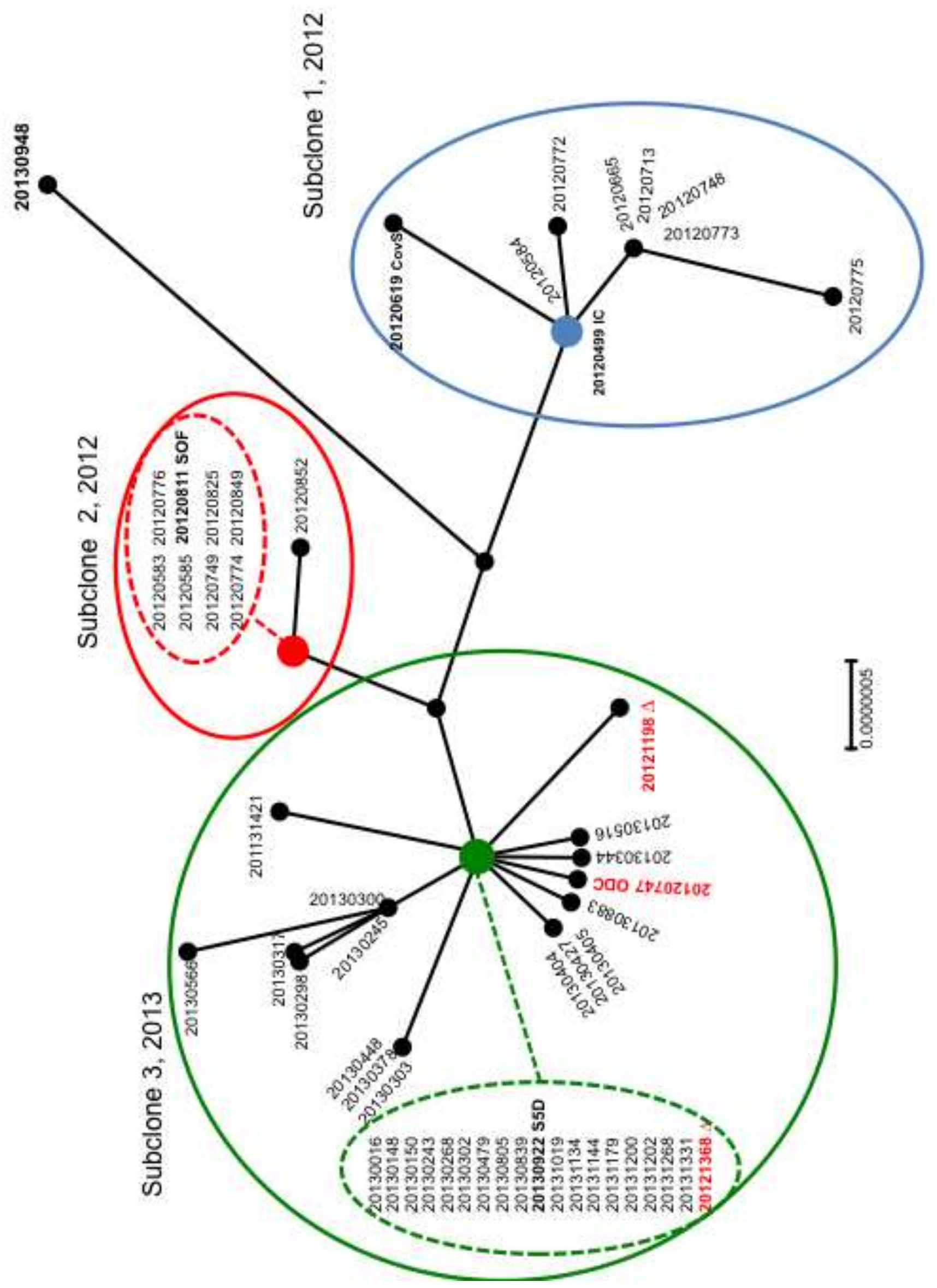




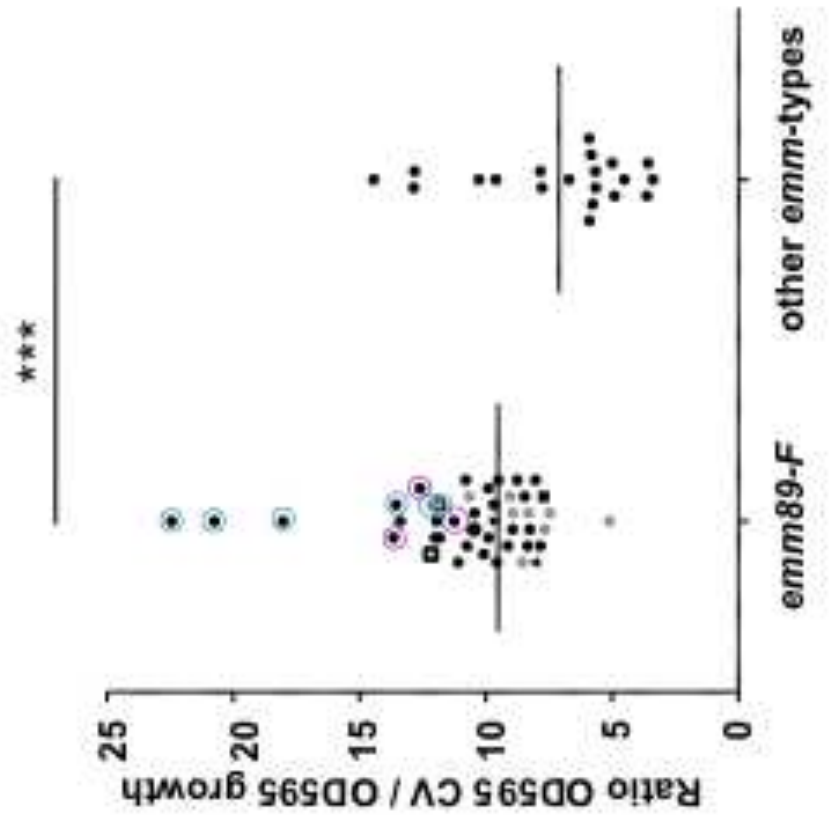




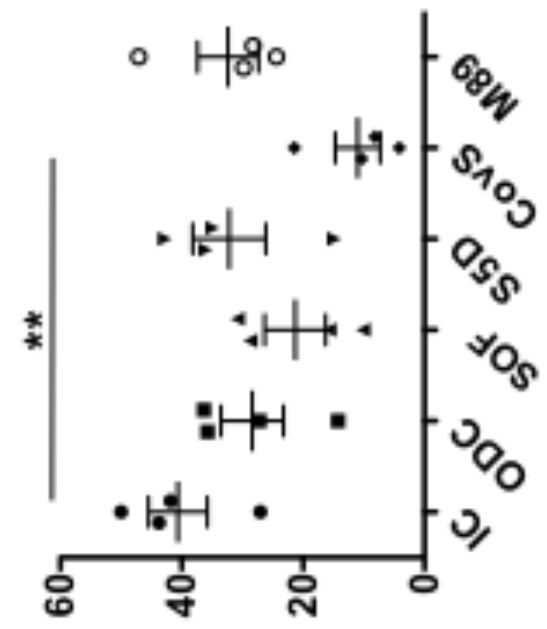

ןen!̣uns \%

๓

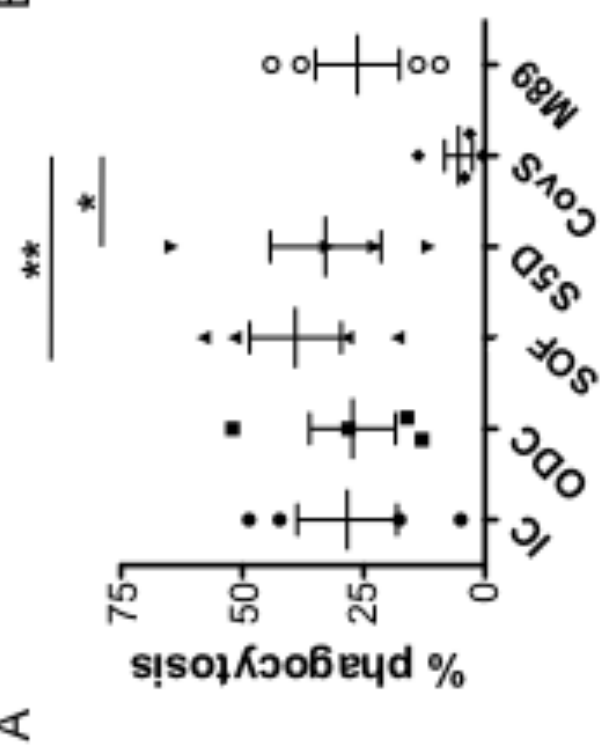


Table S1. Clinical and microbiological characteristics of Streptococcus pyogenes strains isolated during the outbreak

\begin{tabular}{|c|c|c|c|c|c|c|c|c|c|c|c|c|c|c|}
\hline Strain & $\begin{array}{l}\text { Isolation } \\
\text { Date }\end{array}$ & Age & Sexe & $\begin{array}{l}\text { Anatomical origin / } \\
\text { Patient initials a }\end{array}$ & $\begin{array}{l}\text { Infection } \\
\text { status }\end{array}$ & Death & $\begin{array}{l}\text { Tracheos } \\
\text { tomy } \\
\text { cannula }\end{array}$ & HCW & $\begin{array}{l}\text { Molecular } \\
\text { markers }\end{array}$ & $\begin{array}{l}\text { emm } \\
\text { genotype }\end{array}$ & $\begin{array}{l}\text { PFGE } \\
\text { pattern }\end{array}$ & $\mathbf{A T B}^{\mathbf{b}}$ & WGS & $\begin{array}{l}\text { biofilm / } \\
\text { cellular } \\
\text { analysis }\end{array}$ \\
\hline 20120499 & $16 / 04 / 12$ & 58 & $\mathrm{M}$ & Blood culture & Invasive & & & & speB, speC, smeZ & emm89 & 89-A4 & $\mathrm{S}$ & + & $\mathrm{IC}$ \\
\hline 20120583 & $04 / 05 / 12$ & 65 & M & Blood culture & Invasive & & + & & speB, spec, smeZ & emm89 & $89-A 4$ & S & + & + \\
\hline 20120584 & $03 / 05 / 12$ & 64 & $\mathrm{~F}$ & Blood culture & Invasive & + & & & speB, speC, smeZ & emm89 & $89-\mathrm{A} 4$ & S & + & + \\
\hline 20120585 & $07 / 05 / 12$ & 56 & M & Blood culture & Invasive & & & & speB, speC, smeZ & emm89 & $89-\mathrm{A} 4$ & S & + & + \\
\hline 20120619 & $14 / 05 / 12$ & 27 & $\mathrm{~F}$ & Pharynx & Colonization & & & + & speB, speC, smeZ & emm89 & $89-\mathrm{A} 4$ & $\mathrm{~S}$ & + & CovS \\
\hline 20120665 & $16 / 05 / 12$ & 53 & M & Gastrostomy site & Non-invasive & & & & speB, speC, smeZ & emm89 & $89-\mathrm{A} 4$ & S & + & \\
\hline 20120686 & $22 / 05 / 12$ & 35 & $\mathrm{M}$ & Pharynx & Non-invasive & & & & speB, smeZ & emm89 & $89-A$ & $\mathrm{~S}$ & & \\
\hline 20120691 & $18 / 05 / 12$ & 30 & $\mathrm{~F}$ & Pharynx & Non-invasive & & & + & speA, speB, speC, smeZ & emm6 & & S & & + \\
\hline 20120713 & $26 / 05 / 12$ & 65 & M & Pharynx & Colonization & & & & speB, speC, smeZ & emm89 & $89-\mathrm{A} 4$ & S & + & + \\
\hline 20120727 & $29 / 05 / 12$ & 68 & M & Sputum & Colonization & & & & speB, speC, smeZ & emm89 & $89-\mathrm{A} 4$ & S & & \\
\hline 20120747 & $02 / 06 / 12$ & 55 & M & Pharynx & Colonization & & + & & speB, speC, smeZ & emm89 & $89-\mathrm{A} 4$ & $\mathrm{~S}$ & + & ODC \\
\hline 20120748 & $05 / 06 / 12$ & 64 & M & Pharynx & Colonization & & & & speB, speC, smeZ & emm89 & 89-A14 & $\mathrm{TcR}$ & + & \\
\hline 20120749 & $02 / 06 / 12$ & 61 & M & $\begin{array}{l}\text { Protected specimen } \\
\text { brush }\end{array}$ & Invasive & & + & & speB, speC, smeZ & emm89 & $89-\mathrm{A} 4$ & $\mathrm{~S}$ & + & + \\
\hline 20120772 & $09 / 06 / 12$ & 38 & $\mathrm{~F}$ & Pharynx & Non-invasive & & & + & speB, speC, smeZ & emm89 & $89-\mathrm{A} 4$ & S & + & \\
\hline 20120773 & $07 / 06 / 12$ & 60 & M & Pharynx & Colonization & & & & speB, speC, smeZ & emm89 & $89-\mathrm{A} 21$ & TcR & + & \\
\hline 20120774 & $06 / 06 / 12$ & 64 & $\mathrm{~F}$ & Gastrostomy site & Non-invasive & & & & speB, speC, smeZ & emm89 & 89-A4 & $\mathrm{S}$ & + & \\
\hline 20120775 & $05 / 06 / 12$ & 37 & $\mathrm{~F}$ & Pharynx & Non-invasive & & & + & speB, speC, smeZ & emm89 & $89-\mathrm{A} 4$ & S & + & \\
\hline 20120776 & $11 / 06 / 12$ & 27 & $\mathrm{~F}$ & Pharynx & Non-invasive & & & + & speB, speC, smeZ & emm89 & 89-A4 & S & + & + \\
\hline 20120811 & $14 / 06 / 12$ & 38 & $\mathrm{M}$ & Blood culture & Invasive & + & & & speB, speC, smeZ & emm89 & $89-\mathrm{A} 4$ & S & + & FBP \\
\hline 20120824 & $21 / 06 / 12$ & 42 & $\mathrm{~F}$ & Pharynx & Colonization & & & + & speB, speC, smeZ & emm89 & $89-\mathrm{A} 4$ & S & & \\
\hline 20120825 & $24 / 06 / 12$ & 52 & M & Sputum & Colonization & & + & & speB, speC, smeZ & emm89 & 89-A4 & $\mathrm{S}$ & + & + \\
\hline 20120849 & $29 / 06 / 12$ & 58 & M & $\begin{array}{l}\text { Protected specimen } \\
\text { brush }\end{array}$ & Invasive & & + & & speB, speC, smeZ & emm89 & $89-\mathrm{A} 4$ & S & + & \\
\hline 20120852 & $28 / 06 / 12$ & 56 & M & Tracheostomy cannula & Non-invasive & & + & & speB, speC, smeZ & emm89 & $89-\mathrm{A} 4$ & $\mathrm{~S}$ & + & + \\
\hline 20121026 & $02 / 08 / 12$ & 81 & M & Wound & Non-invasive & & + & & speB, speC, smeZ & emm89 & $89-\mathrm{A} 4$ & $\mathrm{~S}$ & & \\
\hline 20121198 & $08 / 09 / 12$ & 64 & M & Blood culture & Invasive & & + & & speB, speC, smeZ & emm89 & $89-A$ & $\mathrm{~S}$ & + & + \\
\hline 20121368 & $20 / 10 / 12$ & 55 & M & Sputum & Non-invasive & & & & speB, smeZ & emm89 & 89-A & S & + & + \\
\hline 20130016 & $14 / 12 / 12$ & 64 & M & $\begin{array}{l}\text { Protected specimen } \\
\text { brush }\end{array}$ & Invasive & & & & speB, speC, smeZ & emm89 & $89-\mathrm{A} 4$ & $\mathrm{~S}$ & + & + \\
\hline 20130148 & $25 / 01 / 13$ & 63 & M & $\begin{array}{l}\text { Protected specimen } \\
\text { brush }\end{array}$ & Invasive & & + & & speB, speC, smeZ & emm89 & 89-A4 & S & + & + \\
\hline 20130149 & $25 / 01 / 13$ & 49 & M & Adenopathy discharge & Non-invasive & & + & & speB, speC, smeZ & emm89 & $89-\mathrm{A} 4$ & S & & \\
\hline 20130150 & $25 / 01 / 13$ & 73 & M & Blood culture & Invasive & & + & & speB, speC, smeZ & emm89 & $89-\mathrm{A} 4$ & $\mathrm{~S}$ & + & + \\
\hline 20130243 & $12 / 02 / 13$ & 60 & M & Blood culture & Invasive & + & & & speB, speC, smeZ & emm89 & 89-A4 & S & + & + \\
\hline 20130244 & $11 / 02 / 13$ & 50 & M & $\begin{array}{l}\text { Protected specimen } \\
\text { brush }\end{array}$ & Invasive & & + & & speB, speC, smeZ & emm89 & $89-\mathrm{A} 4$ & $\mathrm{~S}$ & & \\
\hline 20130245 & $11 / 02 / 13$ & 64 & M & Gastrostomy site & Non-invasive & & & & speB, speC, smeZ & emm89 & $89-\mathrm{A} 4$ & $\mathrm{~S}$ & + & + \\
\hline 20130246 & $11 / 02 / 13$ & 50 & M & Protected specimen & Invasive & & + & & speB, speC, smeZ & emm89 & $89-\mathrm{A} 4$ & S & & \\
\hline
\end{tabular}




\begin{tabular}{|c|c|c|c|c|c|c|c|c|c|c|c|c|c|c|}
\hline & & & & brush & & & & & & & & & & \\
\hline 20130267 & $13 / 02 / 13$ & 65 & $\mathrm{~F}$ & $\mathrm{DHN} / \mathrm{CE}$ & Invasive & & & & speB, smeZ & emm104 & $104-A$ & $\mathrm{TcR}$ & & \\
\hline 20130268 & $15 / 02 / 13$ & 83 & $\mathrm{~F}$ & DHN & Invasive & & & & speB, speC, smeZ & emm89 & $89-\mathrm{A} 4$ & $\mathrm{~S}$ & + & + \\
\hline 20130269 & $15 / 02 / 13$ & 58 & M & Tracheostomy cannula & Colonization & & + & & speB, speC, smeZ & emm89 & $89-\mathrm{A} 4$ & S & & \\
\hline 20130270 & $15 / 02 / 13$ & 52 & $\mathrm{~F}$ & Pharynx & Non-invasive & & & & speB, smeZ & emm104 & $104-\mathrm{A}$ & TcR & & \\
\hline 20130271 & $15 / 02 / 13$ & 68 & M & $\begin{array}{l}\text { Protected specimen } \\
\text { brush }\end{array}$ & Invasive & & & & speB, speC, smeZ & emm89 & $89-\mathrm{A} 4$ & $\mathrm{~S}$ & & \\
\hline 20130298 & $20 / 02 / 13$ & 66 & $\mathrm{~F}$ & Tracheostomy cannula & Non-invasive & & + & & speB, speC, smeZ & emm89 & $89-\mathrm{A} 4$ & $\mathrm{~S}$ & + & + \\
\hline 20130299 & $19 / 02 / 13$ & 84 & M & Wound & Non-invasive & & & & speB, speC, smeZ & emm89 & $89-\mathrm{A} 4$ & S & & \\
\hline 20130300 & $19 / 02 / 13$ & 49 & M & Wound & Non-invasive & & & & speB, speC, smeZ & emm89 & $89-\mathrm{A} 4$ & S & + & + \\
\hline 20130301 & $19 / 02 / 13$ & 54 & M & Wound & Non-invasive & & & & speB, speC, smeZ & emm89 & $89-\mathrm{A} 4$ & S & & \\
\hline 20130302 & $19 / 02 / 13$ & 65 & M & Pharynx & Non-invasive & & & & speB, speC, smeZ & emm89 & $89-\mathrm{A} 4$ & S & + & + \\
\hline 20130303 & $20 / 02 / 13$ & 60 & M & $\begin{array}{l}\text { Tracheostomy } \\
\text { cannula/BP }\end{array}$ & Non-invasive & & + & & speB, speC, smeZ & eтm89 & $89-\mathrm{A} 4$ & $\mathrm{~S}$ & + & + \\
\hline 20130316 & $25 / 02 / 13$ & 72 & M & $\begin{array}{l}\text { Protected specimen } \\
\text { brush }\end{array}$ & Invasive & & + & & speB, speC, smeZ & emm89 & $89-\mathrm{A} 4$ & S & & \\
\hline 20130317 & $22 / 02 / 13$ & 59 & M & Tracheostomy cannula & Non-invasive & & + & & speB, speC, smeZ & eтm89 & $89-\mathrm{A} 4$ & S & + & + \\
\hline 20130318 & $24 / 02 / 13$ & 69 & M & $\begin{array}{l}\text { Protected specimen } \\
\text { brush }\end{array}$ & Invasive & + & + & & speB, speC, smeZ & emm89 & $89-\mathrm{A} 4$ & $\mathrm{~S}$ & & \\
\hline 20130319 & $25 / 02 / 13$ & 23 & $\mathrm{~F}$ & Pharynx & Non-invasive & & & + & speB, speC, smeZ & eтm89 & $89-A 4$ & $\mathrm{~S}$ & & \\
\hline 20130344 & $04 / 03 / 13$ & 73 & $\mathrm{~F}$ & Wound & Non-invasive & & & & speB, speC, smeZ & emm89 & $89-\mathrm{A} 4$ & $\mathrm{~S}$ & + & + \\
\hline 20130365 & $06 / 03 / 13$ & 74 & $\mathrm{f}$ & Wound & Colonization & & & & speB, speC, smeZ & emm89 & $89-\mathrm{A} 4$ & S & & \\
\hline 20130377 & $12 / 03 / 13$ & 24 & $\mathrm{~m}$ & Pharynx & Non-invasive & & + & & speB, speC, smeZ & emm75 & & S & & \\
\hline 20130378 & $11 / 03 / 13$ & 60 & $\mathrm{~m}$ & $\begin{array}{l}\text { Tracheostomy } \\
\text { cannula/BP }\end{array}$ & Non-invasive & & + & & speB, speC, smeZ & emm89 & $89-\mathrm{A} 4$ & $\mathrm{~S}$ & + & + \\
\hline 20130403 & $18 / 03 / 13$ & 66 & $\mathrm{~F}$ & Wound/CE & Colonization & & & & speB, smeZ & emm104 & & $\mathrm{TcR}$ & & \\
\hline 20130404 & $20 / 03 / 13$ & 22 & M & Pharynx & Non-invasive & & & + & speB, speC, smeZ & emm89 & $89-\mathrm{A} 4$ & $\mathrm{~S}$ & + & + \\
\hline 20130405 & $19 / 03 / 13$ & 21 & $\mathrm{~F}$ & Pharynx & Non-invasive & & & + & speB, speC, smeZ & emm89 & $89-\mathrm{A} 4$ & S & + & + \\
\hline 20130427 & $17 / 03 / 13$ & 87 & M & $\begin{array}{l}\text { Protected specimen } \\
\text { brush }\end{array}$ & Invasive & & + & & speB, speC, smeZ & emm89 & $89-\mathrm{A} 4$ & S & + & + \\
\hline 20130448 & $03 / 04 / 13$ & 61 & M & $\begin{array}{l}\text { Tracheostomy } \\
\text { cannula/BP }\end{array}$ & Non-invasive & & + & & speB, speC, smeZ & emm89 & $89-\mathrm{A} 4$ & $\mathrm{~S}$ & + & \\
\hline 20130479 & $08 / 04 / 13$ & 78 & M & Blood culture & Invasive & & + & & speB, speC, smeZ & emm89 & $89-\mathrm{A} 4$ & $\mathrm{~S}$ & + & + \\
\hline 20130516 & $15 / 04 / 13$ & 53 & M & $\begin{array}{l}\text { Protected specimen } \\
\text { brush }\end{array}$ & Invasive & + & + & & speB, speC, smeZ & emm89 & $89-\mathrm{A} 4$ & $\mathrm{~S}$ & + & + \\
\hline 20130566 & $30 / 04 / 13$ & 61 & M & Wound & Non-invasive & & & & speB, speC, smeZ & emm89 & $89-\mathrm{A} 4$ & S & + & + \\
\hline 20130587 & $06 / 05 / 13$ & 69 & M & Wound & Non-invasive & & & & speB, speC, ssa, smeZ & emm4 & & S & & \\
\hline 20130805 & $01 / 07 / 13$ & 65 & M & Sputum & Non-invasive & & & & speB, speC, smeZ & emm89 & $89-\mathrm{A} 4$ & S & + & + \\
\hline 20130839 & $12 / 07 / 13$ & 28 & $\mathrm{~F}$ & Pharynx/GV & Non-invasive & & & + & speB, speC, smeZ & emm89 & $89-\mathrm{A} 4$ & S & + & + \\
\hline 20130882 & $20 / 07 / 13$ & 80 & M & Gastrostomy site & Non-invasive & & + & & speB, smeZ & emm89 & $89-A$ & S & & \\
\hline 20130883 & $22 / 07 / 13$ & 60 & M & Sputum & Colonization & & + & & speB, speC, smeZ & emm89 & $89-\mathrm{A} 4$ & S & + & + \\
\hline 20130922 & $30 / 07 / 13$ & 74 & M & Blood culture & Invasive & & & & speB, speC, smeZ & emm89 & $89-\mathrm{A} 4$ & S & + & S5D \\
\hline 20130948 & $10 / 08 / 13$ & NA & M & Gastrostomy site & Non-invasive & & & & speB, speC, smeZ & emm89 & $89-\mathrm{A} 4$ & $\mathrm{~S}$ & + & + \\
\hline 20131019 & $28 / 08 / 13$ & 56 & $\mathrm{~F}$ & Gastrostomy site & Non-invasive & & & & speB, speC, smeZ & emm89 & $89-\mathrm{A} 4$ & S & + & \\
\hline 20131020 & $29 / 08 / 13$ & 75 & M & Wound & Non-invasive & & & & speB, smeZ & emm89 & $89-A$ & S & & \\
\hline
\end{tabular}




\begin{tabular}{|c|c|c|c|c|c|c|c|c|c|c|c|c|c|}
\hline 20131102 & $17 / 09 / 13$ & 65 & M & Wound & Non-invasive & & & speB, speC, smeZ & emm89 & $89-\mathrm{A} 4$ & $\mathrm{~S}$ & & \\
\hline 20131103 & $17 / 09 / 13$ & 73 & M & Gastrostomy site & Non-invasive & & & speB, speC, smeZ & emm89 & $89-\mathrm{A} 4$ & $\mathrm{~S}$ & + & + \\
\hline 20131134 & $23 / 09 / 13$ & 28 & $\mathrm{~F}$ & Pharynx/GV & Colonization & & + & speB, speC, smeZ & emm89 & $89-\mathrm{A} 4$ & $\mathrm{~S}$ & + & + \\
\hline 20131144 & $27 / 09 / 13$ & 26 & $\mathrm{~F}$ & Pharynx & Non-invasive & & + & speB, speC, smeZ & emm89 & $89-\mathrm{A} 4$ & $\mathrm{~S}$ & + & + \\
\hline 20131179 & $06 / 10 / 13$ & 61 & M & Blood culture/BP & Invasive & & & speB, speC, smeZ & emm89 & $89-\mathrm{A} 4$ & $\mathrm{~S}$ & + & + \\
\hline 20131188 & $12 / 10 / 13$ & 63 & M & Sputum & Colonization & & & speB, speC, smeZ & emm89 & $89-\mathrm{A} 4$ & $\mathrm{~S}$ & & \\
\hline 20131200 & $15 / 10 / 13$ & 54 & M & Pharynx & Colonization & & & speB, speC, smeZ & emm89 & $89-\mathrm{A} 4$ & $\mathrm{~S}$ & + & + \\
\hline 20131201 & $16 / 10 / 13$ & 36 & $\mathrm{~F}$ & Pharynx & Colonization & & + & speB, speC, ssa, smeZ & emm87 & & $\mathrm{S}$ & & \\
\hline 20131202 & $17 / 10 / 13$ & 74 & $\mathrm{~F}$ & Blood culture & Invasive & & & speB, speC, smeZ & emm89 & $89-\mathrm{A} 4$ & $\mathrm{~S}$ & + & \\
\hline 20131268 & $08 / 11 / 13$ & 54 & M & Gastrostomy site & Colonization & & & speB, speC, smeZ & emm89 & $89-\mathrm{A} 4$ & $\mathrm{~S}$ & + & \\
\hline 20131331 & $25 / 11 / 13$ & 55 & M & Blood culture & Invasive & & & speB, speC, smeZ & emm89 & $89-\mathrm{A} 4$ & $\mathrm{~S}$ & + & \\
\hline 20131380 & $09 / 12 / 13$ & 73 & $\mathrm{~F}$ & Sputum & Colonization & & & speB, speC, smeZ & emm89 & $89-A 4$ & $\mathrm{~S}$ & & \\
\hline 20131421 & $23 / 12 / 13$ & 41 & $\mathrm{M}$ & Tracheostomy cannula & Colonization & + & & speB, speC, smeZ & emm89 & 89-A4 & $\mathrm{S}$ & + & + \\
\hline
\end{tabular}

${ }^{a}$ The patient's initials, in bold, was added when more than one strain was collected from the same individual

${ }^{\mathrm{b}} \mathrm{S}$, susceptible to all antibiotic tested; TcR, tetracycline resistant

${ }^{c}+=$ biofilm formation capacity studied; initials refer to the strains whose interactions with A549 and THP1 have been further studied 
Table S2. Control strains used

\begin{tabular}{|c|c|c|c|c|}
\hline $\begin{array}{l}\text { CNR } \\
\text { Strain } \\
\text { number }\end{array}$ & $\begin{array}{l}\text { Anatomical } \\
\text { origin }\end{array}$ & $\begin{array}{l}\text { emm } \\
\text { genotype }\end{array}$ & $\begin{array}{l}\text { Molecular } \\
\text { markers a }\end{array}$ & Antibiotic resistance ${ }^{b}$ \\
\hline 20120456 & Blood culture & emm89 & speB, speC & $\mathrm{S}$ \\
\hline 20141462 & $\begin{array}{l}\text { Bronchial } \\
\text { aspiration }\end{array}$ & emm89 & speB, speC & $\mathrm{S}$ \\
\hline 20141463 & Wound & emm89 & speB, speC & $\mathrm{S}$ \\
\hline 201410469 & Wound & emm89 & speB, speC & S \\
\hline 20040562 & Blood culture & emml & speA, speB, speJ & S \\
\hline 20070928 & Blood culture & emml & speA, speB, speC & $\mathrm{S}$ \\
\hline 20071056 & Blood culture & emml & speA, speB & S \\
\hline 20080059 & Blood culture & emml & speA, speB & $\mathrm{S}$ \\
\hline 20080166 & Blood culture & emml & speA, speB & $\mathrm{S}$ \\
\hline 20080176 & Blood culture & emml & speA, speB & S \\
\hline 20080304 & Blood culture & emml & speA, speB & S \\
\hline 20090096 & Blood culture & emml & speA, speB & $\mathrm{S}$ \\
\hline 20090301 & Blood culture & emml & speA, speB & $\mathrm{S}$ \\
\hline 20080406 & Blood culture & emm101.2 & speB & $\mathrm{Tc}^{\mathrm{R}}$ \\
\hline 20090408 & Blood culture & emm2 & speB, speC & $\mathrm{S}$ \\
\hline 20090207 & Blood culture & emm2 & speB, speC & S \\
\hline 20060663 & Knee hygroma & emm 28 & speA, speB & $\mathrm{S}$ \\
\hline 20071042 & Blood culture & emm28 & speA, speB & $\mathrm{Kan}^{\mathrm{R}}, \mathrm{Str}^{\mathrm{R}}, \mathrm{Ery}^{\mathrm{R}}, \mathrm{Cli}^{\mathrm{R}}, \mathrm{Tc}^{\mathrm{R}}$ \\
\hline 20090413 & Blood culture & emm 28 & speB, speC & $\mathrm{S}$ \\
\hline 20090146 & Blood culture & emm 28 & speB, speC & $\mathrm{S}$ \\
\hline 20071057 & Vagina & emm 28 & speB, speC & $\mathrm{Kan}^{\mathrm{R}}, \mathrm{Str}^{\mathrm{R}}, \mathrm{Ery}^{\mathrm{R}}, \mathrm{Cli}^{\mathrm{R}}$ \\
\hline 20090450 & Vagina & emm28 & speB, speC & $\mathrm{S}$ \\
\hline 20090153 & Blood culture & emm3 & speA, speB & $\mathrm{S}$ \\
\hline 20080308 & Blood culture & emm90,2 & speB, speC & $\mathrm{Tc}^{\mathrm{R}}$ \\
\hline
\end{tabular}

${ }^{\text {a }}$ The presence of $s m e Z$ was not assayed.

${ }^{\mathrm{b}} \mathrm{S}$, susceptible to all antibiotics tested. ${ }^{\mathrm{R}}$, resistant; Cli, clindamycin; Ery, erythromycin; Kan, kanamycin; Str, streptomycin; Tc, tetracycline 
Table S3. Relevant mutations occuring in the sequenced isolates

\begin{tabular}{|c|c|c|c|c|c|}
\hline \multirow{4}{*}{\multicolumn{3}{|c|}{$\begin{array}{l}\text { position } \\
\text { type of mutation } \\
\text { mutation } \\
\text { transcription orientation of the gene }\end{array}$}} & \multirow{4}{*}{\begin{tabular}{|l|}
4334 \\
$\mathrm{G} \rightarrow \mathrm{A}$ \\
$\mathrm{G} 16 \mathrm{D}(\mathrm{GGC} \rightarrow \mathrm{G} \underline{\underline{A C}})$ \\
$\rightarrow$ \\
\end{tabular}} & \multirow{3}{*}{\begin{tabular}{|l|}
7957 \\
$\mathrm{C} \rightarrow \mathrm{A}$ \\
$\mathrm{D} 525 \mathrm{Y}(\underline{\mathrm{GAT}} \rightarrow \mathrm{TAT})$ \\
\end{tabular}} & \multirow{2}{*}{\begin{tabular}{|l|}
9031 \\
22 bp x 2 \\
\end{tabular}} \\
\hline & & & & & \\
\hline & & & & & duplication \\
\hline & & & & $\leftarrow$ & $\rightarrow$ \\
\hline Strain name & $\begin{array}{l}\text { mutation } \\
\text { profile }^{a}\end{array}$ & $\begin{array}{l}\text { biofilm / cellular } \\
\text { analysis }\end{array}$ & hypothetical protein & \begin{tabular}{|l|} 
Dipeptide-binding $\mathrm{ABC}$ \\
transporter, periplasmic substrate- \\
binding component DppA \\
(TC 3.A.1.5.2)
\end{tabular} & $\begin{array}{l}\text { Fibronectin-binding } \\
\text { protein }\end{array}$ \\
\hline 20120499 & $\mathrm{AP}$ & IC & & & \\
\hline 20120583 & $\mathrm{AL}$ & Yes & & & \\
\hline 20120584 & $\mathrm{AM}$ & Yes & & & \\
\hline 20120585 & $\mathrm{E}$ & Yes & & & \\
\hline 20120619 & $\mathrm{G}$ & CovS & & & \\
\hline 20120665 & $\mathrm{AK}$ & & & & \\
\hline 20120713 & $\mathrm{AK}$ & Yes & & & \\
\hline 20120747 & AG & ODC & & & \\
\hline 20120748 & AK & & & & \\
\hline 20120749 & $\mathrm{U}$ & Yes & & & $\mathbf{X}$ \\
\hline 20120772 & AP & & & & \\
\hline 20120773 & AK & & & & \\
\hline 20120774 & $\mathrm{AP}$ & & & & \\
\hline 20120775 & $\mathrm{AK}$ & & & & \\
\hline 20120776 & $\mathrm{AP}$ & Yes & & & \\
\hline 20120811 & AN & FBP & & & \\
\hline 20120825 & $\mathrm{~T}$ & Yes & & & $\mathbf{X}$ \\
\hline 20120849 & AP & & & & \\
\hline 20120852 & $\mathrm{AJ}$ & Yes & & & \\
\hline 20121198 & $\mathrm{~K}$ & Yes & & & \\
\hline 20121368 & $\mathrm{~V}$ & Yes & & & \\
\hline 20130016 & $\mathrm{AF}$ & Yes & & & \\
\hline 20130148 & $\mathrm{AE}$ & Yes & & & \\
\hline 20130150 & $\mathrm{~J}$ & Yes & & & \\
\hline 20130243 & $\mathrm{AO}$ & Yes & & & \\
\hline 20130245 & $\mathrm{R}$ & Yes & & & \\
\hline 20130268 & $\mathrm{AO}$ & & & & \\
\hline 20130298 & $\mathrm{Q}$ & Yes & & & \\
\hline 20130300 & $\mathrm{~S}$ & Yes & & & \\
\hline 20130302 & $\mathrm{AD}$ & Yes & & & \\
\hline 20130303 & $\mathrm{I}$ & Yes & & & \\
\hline 20130317 & $\mathrm{P}$ & Yes & & & \\
\hline 20130344 & $\mathrm{~L}$ & Yes & & & \\
\hline 20130378 & $\mathrm{H}$ & Yes & & & \\
\hline 20130404 & $Z$ & Yes & & & \\
\hline 20130405 & $\mathrm{Y}$ & Yes & & & \\
\hline 20130427 & $Z$ & Yes & & & \\
\hline 20130448 & $\mathrm{I}$ & & & & \\
\hline 20130479 & $\mathrm{AH}$ & Yes & & & \\
\hline 20130516 & $\mathrm{~W}$ & Yes & $\mathbf{X}$ & & \\
\hline
\end{tabular}




\begin{tabular}{|c|c|c|c|c|c|}
\hline 20130566 & D & Yes & & & \\
\hline 20130805 & AQ & Yes & & & \\
\hline 20130839 & $\mathrm{AH}$ & Yes & & & \\
\hline 20130883 & $\mathrm{AB}$ & Yes & & & \\
\hline 20130922 & $\mathrm{X}$ & S5D & & & \\
\hline 20130948 & $\mathrm{~F}$ & Yes & & & \\
\hline 20131019 & $\mathrm{AH}$ & & & & \\
\hline 20131103 & - & Yes & & & \\
\hline 20131134 & $\mathrm{C}$ & Yes & & & \\
\hline 20131144 & A & Yes & & & \\
\hline 20131179 & B & Yes & & & \\
\hline 20131200 & $\mathrm{AO}$ & Yes & & & \\
\hline 20131202 & A & Yes & & & \\
\hline 20131268 & A & & & & \\
\hline 20131331 & A & & & & \\
\hline 20131421 & AA & Yes & & & \\
\hline
\end{tabular}

${ }^{a}$ The profile is not indicated for the strains outside the epidemic clone

$\mathrm{b}$ yes, biofilm formation assayed; strains further analyzed are indicated by their acronymes 


\begin{tabular}{|c|c|c|c|}
\hline 13953 & 14733 & 21044 & 21074 \\
\hline$\Delta 1 \mathrm{bp}$ & $\mathrm{C} \rightarrow \mathrm{T}$ & $11 \mathrm{bp} \times 2$ & $\mathrm{C} \rightarrow \mathrm{A}$ \\
\hline \begin{tabular}{|c|}
$\operatorname{coding}(189 / 1284 \mathrm{nt})$ \\
\end{tabular} & $\mathrm{A} 142 \mathrm{~T}(\mathrm{GCC} \rightarrow \mathrm{ACC})$ & duplication & $\mathrm{A} 38 \mathrm{D}(\mathrm{GCC} \rightarrow \mathrm{GAC})$ \\
\hline$\leftarrow$ & $\leftarrow$ & $\rightarrow$ & $\rightarrow$ \\
\hline $\begin{array}{l}\text { FIG009439: Cytosolic protein } \\
\text { containing multiple CBS } \\
\text { domains }\end{array}$ & $\begin{array}{l}\text { L-xylulose 5-phosphate 3- } \\
\text { epimerase (EC 5.1.3.--) }\end{array}$ & $\begin{array}{l}\text { Transmembrane histidine kinase } \\
\text { CovS }\end{array}$ & $\begin{array}{l}\text { Transmembrane histidine kinase } \\
\text { CovS }\end{array}$ \\
\hline & & & \\
\hline & & & \\
\hline & & & \\
\hline & & $\mathbf{X}$ & \\
\hline & & & \\
\hline & & & \\
\hline & & & \\
\hline & & & \\
\hline & & & \\
\hline & & & \\
\hline & & & \\
\hline & & & \\
\hline & & & \\
\hline & & & \\
\hline & & & \\
\hline & & & \\
\hline & & & \\
\hline & & & \\
\hline & & & \\
\hline & & & \\
\hline $\mathbf{X}$ & & & \\
\hline & & & \\
\hline & & & \\
\hline & & & \\
\hline & & & \\
\hline & & & \\
\hline & & & \\
\hline & & & \\
\hline & & & \\
\hline & & & \\
\hline & & & $\mathbf{X}$ \\
\hline & & & \\
\hline & & & $\mathbf{X}$ \\
\hline & & & \\
\hline & & & \\
\hline & & & \\
\hline & & & $\mathbf{X}$ \\
\hline & & & \\
\hline & & & \\
\hline
\end{tabular}




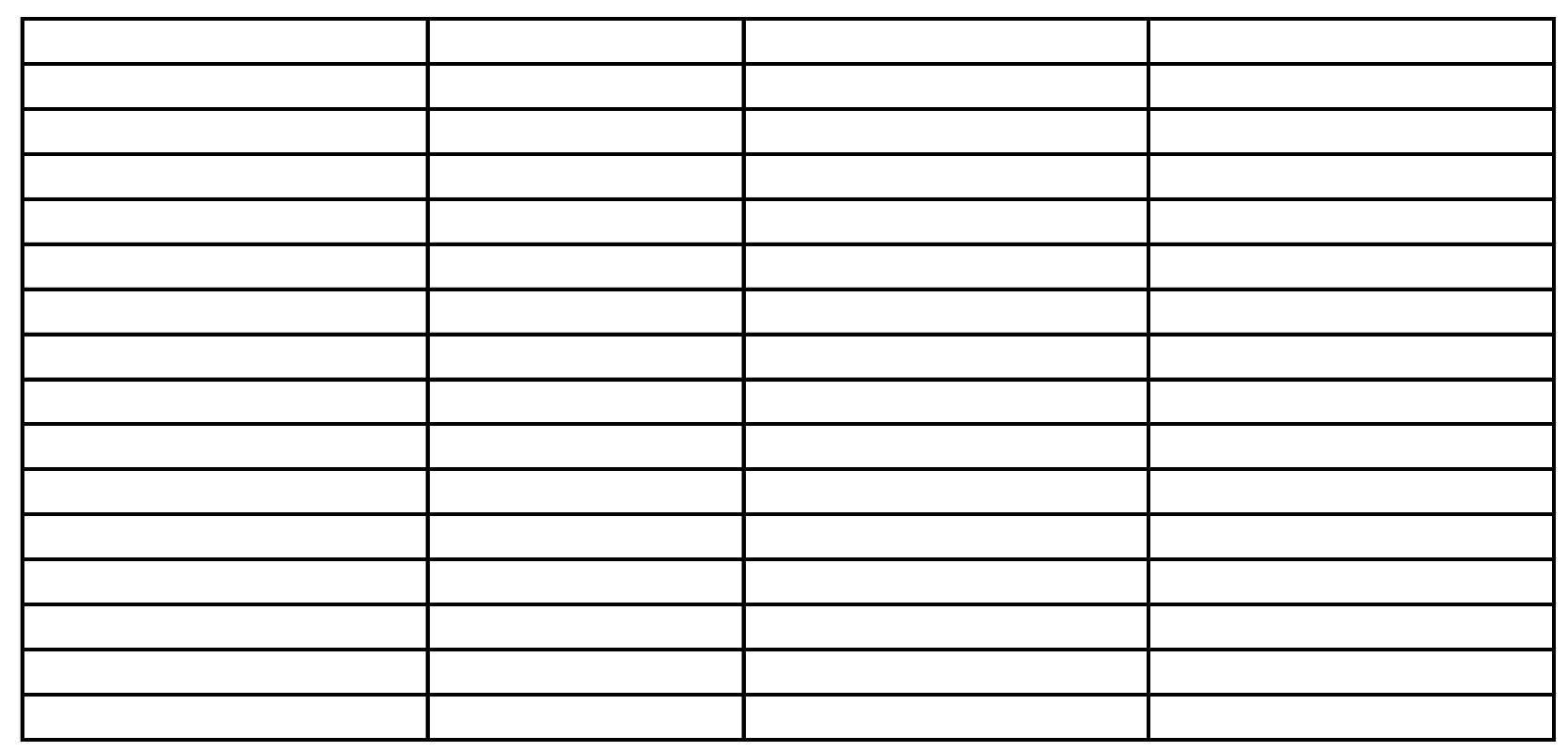




\begin{tabular}{|c|c|c|c|}
\hline 21763 & 21964 & 22055 & 22168 \\
\hline$\Delta 2 \mathrm{bp}$ & $\mathrm{C} \rightarrow \mathrm{T}$ & $A \rightarrow G$ & $\Delta 5 \mathrm{bp}$ \\
\hline coding $(802-803 / 1503 \mathrm{nt})$ & Q335* $(\underline{\mathrm{CAA}} \rightarrow \underline{\mathrm{TAA}})$ & D365G $(\mathrm{GAT} \rightarrow \mathrm{GGT})$ & coding (1207-1211/1503 nt) \\
\hline$\rightarrow$ & $\rightarrow$ & $\rightarrow$ & $\rightarrow$ \\
\hline $\begin{array}{l}\text { Transmembrane histidine kinase } \\
\text { CovS }\end{array}$ & $\begin{array}{l}\text { Transmembrane histidine kinase } \\
\text { CovS }\end{array}$ & $\begin{array}{l}\text { Transmembrane histidine kinase } \\
\text { CovS }\end{array}$ & $\begin{array}{l}\text { Transmembrane histidine kinase } \\
\text { CovS }\end{array}$ \\
\hline & & & \\
\hline & & & \\
\hline & & & \\
\hline & & & \\
\hline & & & $\mathbf{X}$ \\
\hline & & & \\
\hline & & & \\
\hline & & & \\
\hline & & & \\
\hline & & & \\
\hline & & & \\
\hline & & & \\
\hline & & & \\
\hline & & & \\
\hline & & & \\
\hline & & & \\
\hline & & & \\
\hline & & & \\
\hline & & $\mathbf{X}$ & \\
\hline & & & \\
\hline & & & \\
\hline & & & \\
\hline $\mathbf{X}$ & & & \\
\hline & & & \\
\hline & & & \\
\hline & & & \\
\hline & & & \\
\hline & & & \\
\hline & & & \\
\hline & & & \\
\hline & & & \\
\hline & & & \\
\hline & & & \\
\hline & & & \\
\hline & & & \\
\hline & & & \\
\hline & & & \\
\hline & & & \\
\hline & & & \\
\hline
\end{tabular}




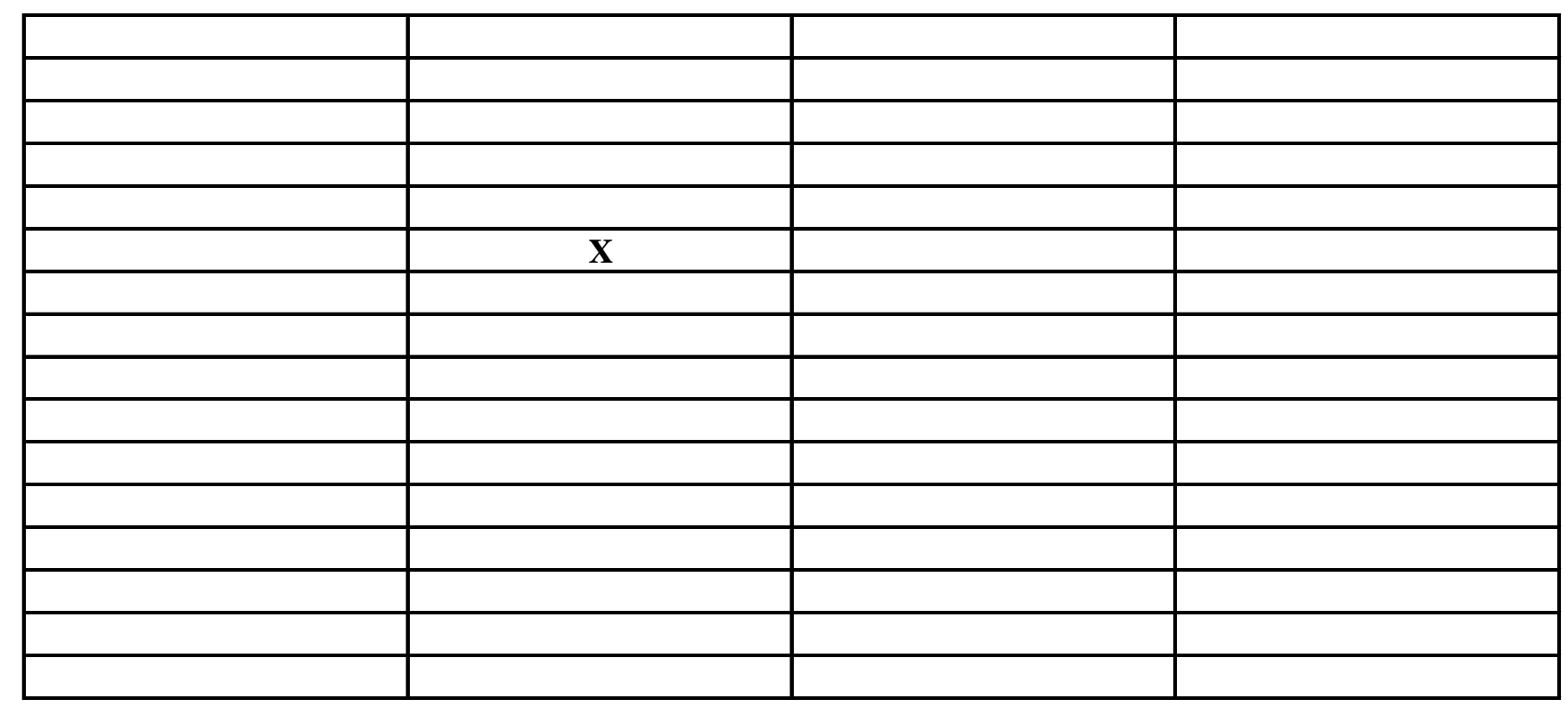




\begin{tabular}{|c|c|c|c|}
\hline 22538 & 37183 & 37290 & 50763 \\
\hline $\mathrm{C} \rightarrow \mathrm{T}$ & $\mathrm{C} \rightarrow \mathrm{T}$ & $\mathrm{G} \rightarrow \mathrm{A}$ & $\mathrm{C} \rightarrow \mathrm{A}$ \\
\hline T147I (ACT $\rightarrow$ ATT $)$ & S79F $(\mathrm{TCC} \rightarrow \mathrm{TTC})$ & $\mathrm{A} 115 \mathrm{~T}(\mathrm{GCT} \rightarrow \mathrm{ACT})$ & D107Y $($ GAT $\rightarrow$ TAT $)$ \\
\hline$\rightarrow$ & $\rightarrow$ & $\rightarrow$ & $\leftarrow$ \\
\hline $\begin{array}{l}\text { Dihydroorotate } \\
\text { dehydrogenase, catalytic } \\
\text { subunit } \\
\text { (EC 1.3.3.1) }\end{array}$ & $\begin{array}{l}\text { Topoisomerase IV subunit } \\
\text { A } \quad \text { (EC 5.99.1.- })\end{array}$ & $\begin{array}{l}\text { Topoisomerase IV subunit } \\
\text { A (EC 5.99.1.- })\end{array}$ & $\begin{array}{l}\text { Response regulator of the } \\
\text { competence regulon ComE }\end{array}$ \\
\hline & & & \\
\hline & & & \\
\hline & & & \\
\hline & & & \\
\hline & & & \\
\hline & & & \\
\hline & & & \\
\hline $\mathbf{X}$ & & & \\
\hline & & & \\
\hline & & & \\
\hline & & & \\
\hline & & & \\
\hline & & & \\
\hline & & & \\
\hline & & & \\
\hline & & & \\
\hline & & & \\
\hline & & & \\
\hline & & & \\
\hline & & & \\
\hline & & & \\
\hline & & & \\
\hline & & & \\
\hline & & & \\
\hline & & & \\
\hline & & & \\
\hline & & & \\
\hline & & & $\mathbf{X}$ \\
\hline & & & \\
\hline & & & \\
\hline & & & \\
\hline & & & \\
\hline & & & \\
\hline & & & \\
\hline & & & \\
\hline & & & \\
\hline & & & \\
\hline & & & \\
\hline & & & \\
\hline & & & \\
\hline
\end{tabular}




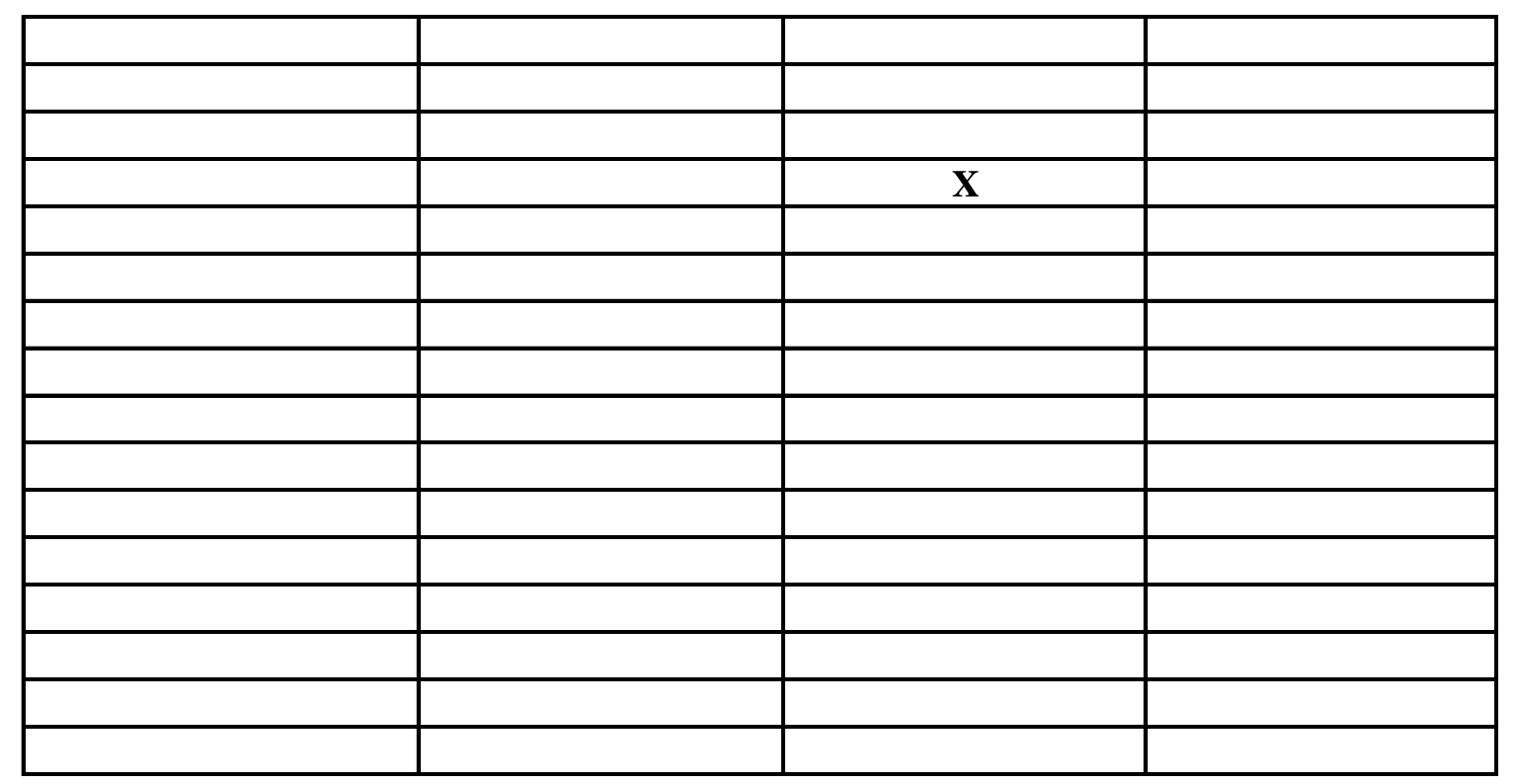




\begin{tabular}{|c|c|c|c|}
\hline 51299 & 60020 & 61356 & 61564 \\
\hline $\mathrm{C} \rightarrow \mathrm{T}$ & 22 bp $\times 2$ & $\mathrm{G} \rightarrow \mathrm{A}$ & $\mathrm{C} \rightarrow \mathrm{A}$ \\
\hline $\mathrm{H} 97 \mathrm{Y}(\underline{\mathrm{CAT}} \rightarrow \underline{\mathrm{TAT}})$ & duplication & $\mathrm{A} 403 \mathrm{~T}(\mathrm{GCG} \rightarrow \underline{\mathrm{ACG}})$ & $\mathrm{A} 472 \mathrm{D}(\mathrm{GCT} \rightarrow \mathrm{GAT})$ \\
\hline$\rightarrow$ & $\rightarrow$ & $\rightarrow$ & $\rightarrow$ \\
\hline $\begin{array}{l}\text { Pyruvate formate-lyase activating } \\
\text { enzyme (EC 1.97.1.4) }\end{array}$ & $\begin{array}{l}\text { Fibronectin-binding } \\
\text { protein }\end{array}$ & $\begin{array}{l}\text { Fibronectin-binding } \\
\text { protein }\end{array}$ & $\begin{array}{l}\text { Fibronectin-binding } \\
\text { protein }\end{array}$ \\
\hline & & & \\
\hline & & & \\
\hline & & & \\
\hline & & & \\
\hline & & $\mathbf{X}$ & \\
\hline & & & $\mathbf{X}$ \\
\hline & & & $\bar{X}$ \\
\hline & & & \\
\hline & & & $\mathbf{X}$ \\
\hline & & & \\
\hline & & & \\
\hline & & & $\mathbf{X}$ \\
\hline & & & $\mathbf{Y}$ \\
\hline & & & $\mathbf{X}$ \\
\hline & $\mathbf{X}$ & & \\
\hline & & & \\
\hline & & & \\
\hline & & & \\
\hline & & & \\
\hline & & & \\
\hline & & & \\
\hline & & & \\
\hline & & & \\
\hline & & & \\
\hline & & & \\
\hline & & & \\
\hline & & & \\
\hline & & & \\
\hline & & & \\
\hline & & & \\
\hline $\mathbf{X}$ & & & \\
\hline & & & \\
\hline & & & \\
\hline & & & \\
\hline & & & \\
\hline & & & \\
\hline & & & \\
\hline & & & \\
\hline
\end{tabular}




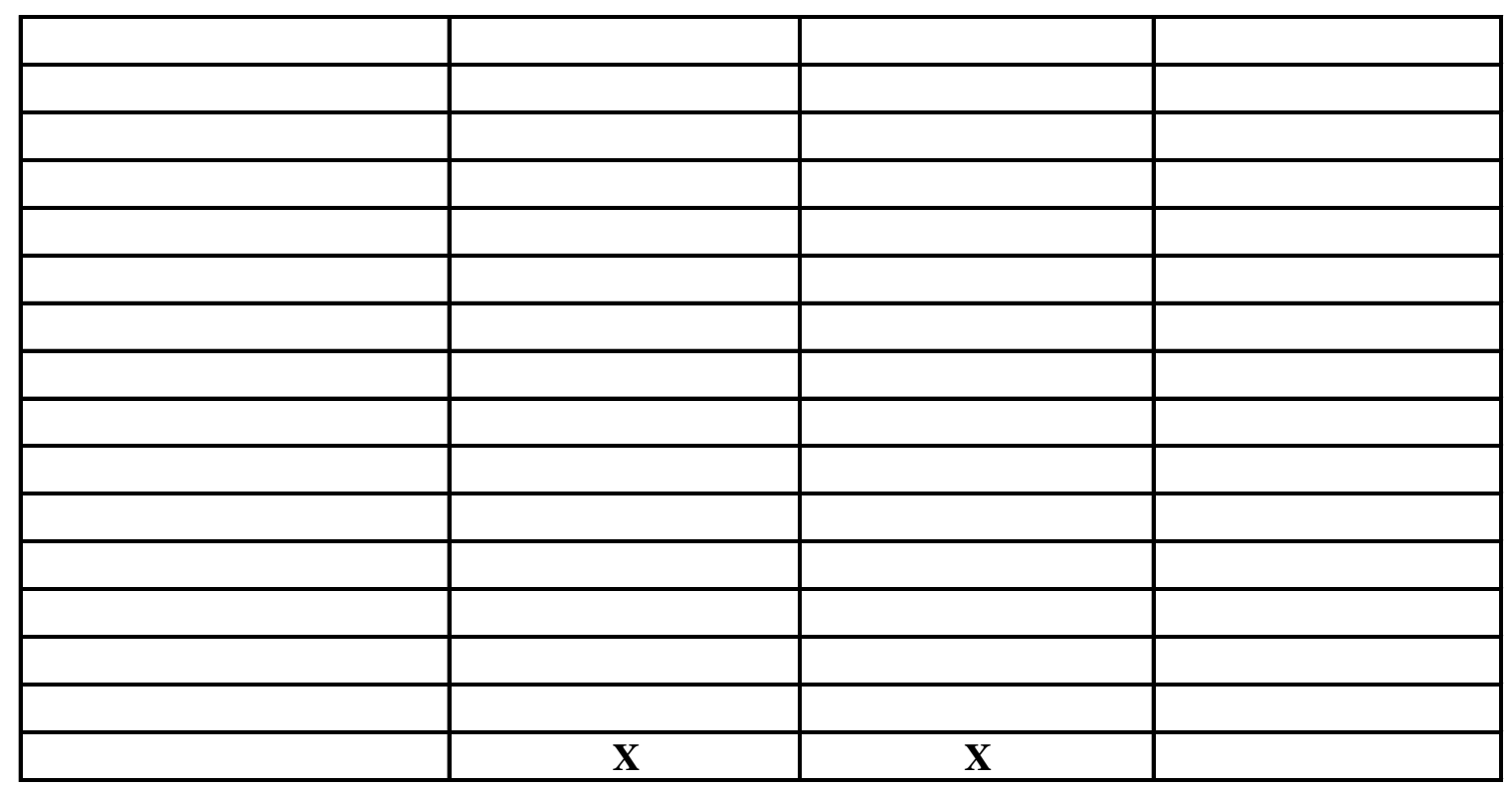




\begin{tabular}{|c|c|c|c|c|}
\hline 61557 & 62662 & 62702 & 62763 & 62796 \\
\hline$\Delta 1 \mathrm{bp}$ & $\mathrm{C} \rightarrow \mathrm{T}$ & $\mathrm{G} \rightarrow \mathrm{T}$ & $\mathrm{G} \rightarrow \mathrm{A}$ & $A \rightarrow G$ \\
\hline coding $(1408 / 2967 \mathrm{nt})$ & $\mathrm{A} 838 \mathrm{~V}(\mathrm{GCA} \rightarrow \mathrm{GTA})$ & $\mathrm{E} 851 \mathrm{D}(\mathrm{GAG} \rightarrow \mathrm{GAT})$ & V872I (GTC $\rightarrow$ ATC) & I883V $(\underline{A T C} \rightarrow \underline{\text { GTC }})$ \\
\hline$\rightarrow$ & $\rightarrow$ & $\rightarrow$ & $\rightarrow$ & $\rightarrow$ \\
\hline \multirow[t]{5}{*}{$\begin{array}{l}\text { Fibronectin-binding } \\
\text { protein }\end{array}$} & $\begin{array}{l}\text { Fibronectin-binding } \\
\text { protein }\end{array}$ & $\begin{array}{l}\text { Fibronectin-binding } \\
\text { protein }\end{array}$ & $\begin{array}{l}\text { Fibronectin-binding } \\
\text { protein }\end{array}$ & $\begin{array}{l}\text { Fibronectin-binding } \\
\text { protein }\end{array}$ \\
\hline & & & $\mathbf{X}$ & \\
\hline & & $\mathbf{X}$ & & $\mathbf{X}$ \\
\hline & & & & \\
\hline & $\mathbf{X}$ & & & \\
\hline & & & & \\
\hline & & & & \\
\hline & & & & \\
\hline & & & & \\
\hline & & & & \\
\hline & & & & \\
\hline & & & & \\
\hline & & & & \\
\hline & & & & \\
\hline & & & & \\
\hline & & & & \\
\hline & & & & \\
\hline & & & & \\
\hline & & & & \\
\hline & & & & \\
\hline & & & $\mathbf{X}$ & $\mathbf{X}$ \\
\hline & & & & \\
\hline & & & & \\
\hline & & & & \\
\hline & & & & \\
\hline & & & & \\
\hline & & & & \\
\hline & & & & \\
\hline & & & & \\
\hline & & & $\mathbf{X}$ & \\
\hline $\mathbf{X}$ & & & & \\
\hline & & & & \\
\hline & & & & \\
\hline & & & & \\
\hline & & & & \\
\hline & & & & \\
\hline & & & & \\
\hline $\mathbf{X}$ & & & & \\
\hline & & & & \\
\hline & & & & \\
\hline
\end{tabular}




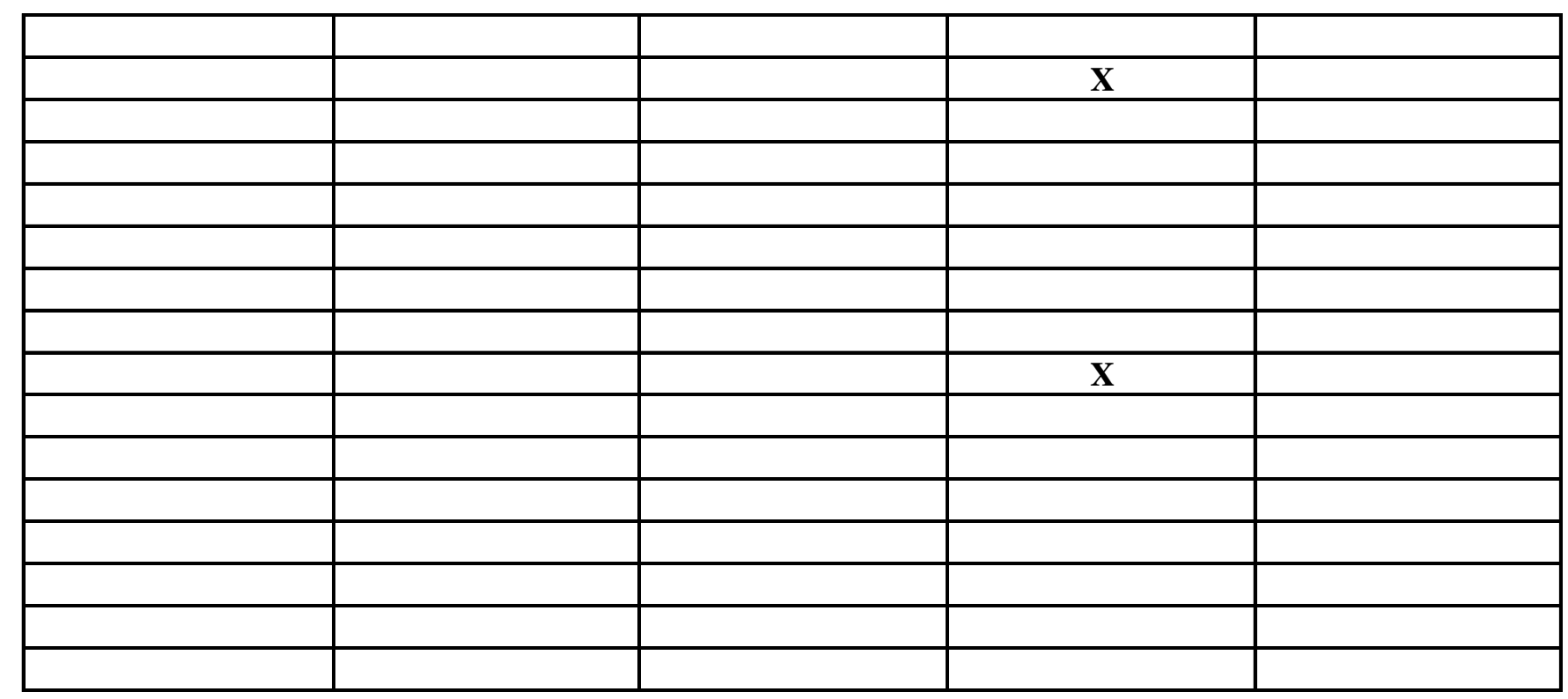




\begin{tabular}{|c|c|c|c|c|}
\hline 62819 & 62850 & 62853 & 70969 & 75410 \\
\hline $\mathrm{T} \rightarrow \mathrm{G}$ & $\mathrm{G} \rightarrow \mathrm{A}$ & $A \rightarrow G$ & $\mathrm{C} \rightarrow \mathrm{T}$ & $\mathrm{G} \rightarrow \mathrm{T}$ \\
\hline $\mathrm{D} 890 \mathrm{E}(\mathrm{GAT} \rightarrow \mathrm{GAG})$ & G901S (GGT $\rightarrow$ AGT) & M902V ( & T10I (ACA $\rightarrow$ ATA $)$ & G278V (GGC $\rightarrow$ GTC $)$ \\
\hline$\rightarrow$ & $\rightarrow$ & $\rightarrow$ & $\rightarrow$ & $\rightarrow$ \\
\hline \multirow[t]{4}{*}{$\begin{array}{l}\text { Fibronectin-binding } \\
\text { protein }\end{array}$} & $\begin{array}{l}\text { Fibronectin-binding } \\
\text { protein }\end{array}$ & $\begin{array}{l}\text { Fibronectin-binding } \\
\text { protein }\end{array}$ & $\begin{array}{l}\text { Thymidine kinase } \\
\text { (EC 2.7.1.21) }\end{array}$ & $\begin{array}{l}\text { M protein trans-acting } \\
\text { positive regulator (Mga) }\end{array}$ \\
\hline & $X$ & $\mathbf{X}$ & & \\
\hline & & & & \\
\hline & $\mathbf{X}$ & $\mathbf{X}$ & & \\
\hline & & & & \\
\hline & & & & \\
\hline & & & & \\
\hline & & & & \\
\hline & & & & \\
\hline & & & & \\
\hline & & & & \\
\hline & & & & \\
\hline & & & & \\
\hline & & & & \\
\hline & & & & \\
\hline & & & & \\
\hline & & & & \\
\hline & & & & \\
\hline & & & & \\
\hline $\mathbf{X}$ & $\mathbf{X}$ & $\mathbf{X}$ & & \\
\hline & $\mathbf{X}$ & $\bar{X}$ & & \\
\hline & $\bar{X}$ & $\mathbf{X}$ & & \\
\hline & & & & \\
\hline & $\mathbf{X}$ & $\mathbf{X}$ & & \\
\hline & & & & \\
\hline & & & & \\
\hline $\mathbf{X}$ & $\mathbf{X}$ & $\mathbf{X}$ & & \\
\hline & & & & \\
\hline & $\mathbf{X}$ & $\mathbf{X}$ & & $\mathbf{X}$ \\
\hline & & & & \\
\hline & & & & \\
\hline & & & & \\
\hline & $\mathbf{X}$ & $\mathbf{X}$ & & \\
\hline & & & & \\
\hline & & & & \\
\hline & $\mathbf{X}$ & $\mathbf{X}$ & & \\
\hline & $\mathbf{X}$ & $\mathbf{X}$ & & \\
\hline
\end{tabular}




\begin{tabular}{|l|l|l|l|l|}
\hline & $\mathbf{X}$ & $\mathbf{X}$ & & \\
\hline $\mathbf{X}$ & & & & \\
\hline & $\mathbf{X}$ & $\mathbf{X}$ & & \\
\hline & & & & \\
\hline & & & & \\
\hline & $\mathbf{X}$ & $\mathbf{X}$ & & \\
\hline & & & $\mathbf{X}$ & \\
\hline & $\mathbf{X}$ & & & \\
\hline $\mathbf{X}$ & $\mathbf{X}$ & $\mathbf{X}$ & & \\
\hline & & & & \\
\hline & & & & \\
\hline & & & & \\
\hline & & & & \\
\hline & & & & \\
\hline & & & & \\
\hline
\end{tabular}




\begin{tabular}{|c|c|c|c|}
\hline 75758 & 78460 & 97446 & 109944 \\
\hline $\mathrm{A} \rightarrow \mathrm{G}$ & $\mathrm{G} \rightarrow \mathrm{A}$ & $\mathrm{T} \rightarrow \mathrm{C}$ & $\mathrm{A} \rightarrow \mathrm{G}$ \\
\hline E394G (GAA $\rightarrow$ GGA) & D178N (GAT $\rightarrow$ AAT $)$ & N193S (AAT $\rightarrow$ AGT) & $\mathrm{D} 274 \mathrm{G}(\mathrm{GAC} \rightarrow \mathrm{GGC})$ \\
\hline$\rightarrow$ & $\rightarrow$ & $\leftarrow$ & $\rightarrow$ \\
\hline $\begin{array}{l}\text { M protein trans-acting } \\
\text { positive regulator (Mga) }\end{array}$ & $\begin{array}{l}\text { Methionyl-tRNA synthetase (EC } \\
6.1 .1 .10)\end{array}$ & $\begin{array}{l}\text { ATP synthase beta } \\
\text { chain } \quad \text { (EC } \\
\text { 3.6.3.14) }\end{array}$ & $\begin{array}{l}\text { Oxaloacetate } \\
\text { decarboxylase beta chain } \\
\text { (EC 4.1.1.3) }\end{array}$ \\
\hline & & & \\
\hline & & & \\
\hline & & & \\
\hline & & & \\
\hline & & & \\
\hline & & & \\
\hline & & & $\mathbf{X}$ \\
\hline & & & \\
\hline & & & \\
\hline & & & \\
\hline & & & \\
\hline & & & \\
\hline & & & \\
\hline & & & \\
\hline & & & \\
\hline & & & \\
\hline & & & \\
\hline & & & \\
\hline & & & $\mathbf{X}$ \\
\hline & & & $\mathbf{X}$ \\
\hline & & & $\bar{X}$ \\
\hline & & & $\mathbf{X}$ \\
\hline & & & $\mathbf{X}$ \\
\hline & & & $\bar{X}$ \\
\hline & & & $\bar{X}$ \\
\hline & & & $\bar{X}$ \\
\hline & & & $\bar{X}$ \\
\hline & & & $\mathbf{X}$ \\
\hline & & & $\mathbf{X}$ \\
\hline & & & $\mathbf{X}$ \\
\hline & & & $\bar{X}$ \\
\hline & & & $\mathbf{X}$ \\
\hline & & & $\mathbf{X}$ \\
\hline & & & $\mathbf{X}$ \\
\hline & & & $\bar{X}$ \\
\hline & & & $\mathrm{X}$ \\
\hline & & & $\mathbf{X}$ \\
\hline & & & $\mathbf{X}$ \\
\hline & & & $\mathbf{X}$ \\
\hline
\end{tabular}




\begin{tabular}{|c|c|c|c|}
\hline $\mathbf{X}$ & & & $\mathbf{X}$ \\
\hline & & & $\mathbf{X}$ \\
\hline & & & $\mathbf{X}$ \\
\hline & & & $\mathbf{X}$ \\
\hline & & & $\mathbf{X}$ \\
\hline & & & $\mathbf{X}$ \\
\hline & & & $\mathbf{X}$ \\
\hline & & & $\mathbf{X}$ \\
\hline & & & $\mathbf{X}$ \\
\hline & & & $\mathbf{X}$ \\
\hline & & & $\mathbf{X}$ \\
\hline & & & $\mathbf{X}$ \\
\hline & & & $\mathbf{X}$ \\
\hline & & & \\
\hline
\end{tabular}




\begin{tabular}{|c|c|c|c|}
\hline \multirow{3}{*}{\begin{tabular}{|l|}
136599 \\
$\Delta 26,400 \mathrm{bp}$ \\
\end{tabular}} & 145637 & 194925 & 249724 \\
\hline & $\Delta 1 \mathrm{bp}$ & $\mathrm{T} \rightarrow \mathrm{A}$ & $\Delta 1 \mathrm{bp}$ \\
\hline & coding $(612 / 636 \mathrm{nt})$ & $\mathrm{S} 296 \mathrm{C}(\mathrm{AGC} \rightarrow \underline{\mathrm{TGC}})$ & coding $(814 / 873 \mathrm{nt})$ \\
\hline []- & $\rightarrow$ & $\leftarrow$ & $\leftarrow$ \\
\hline $\begin{array}{l}34 \text { genes deletion of the } \\
\text { phage containaing speC }\end{array}$ & $\begin{array}{l}\text { Guanylate kinase } \\
\text { (EC 2.7.4.8) }\end{array}$ & $\begin{array}{l}\text { 6-phosphofructokinase } \\
\text { (EC 2.7.1.11) }\end{array}$ & $\begin{array}{l}\text { Shikimate 5- } \\
\text { dehydrogenase I alpha } \\
\text { (EC 1.1.1.25) }\end{array}$ \\
\hline & & & \\
\hline & & & \\
\hline & & & \\
\hline & & & \\
\hline & & & \\
\hline & & & \\
\hline & & & \\
\hline & & & \\
\hline & & & \\
\hline & & & \\
\hline & & & \\
\hline & & & \\
\hline & & & \\
\hline & & & \\
\hline & & & \\
\hline & & & \\
\hline & & & \\
\hline & & & \\
\hline & & & \\
\hline $\mathbf{X}$ & & & \\
\hline $\mathbf{X}$ & & & \\
\hline & & & \\
\hline & & & $\mathbf{X}$ \\
\hline & & & $\mathbf{X}$ \\
\hline & & & $\bar{X}$ \\
\hline & & & $\mathbf{X}$ \\
\hline & & & $\bar{X}$ \\
\hline & & & $\mathbf{X}$ \\
\hline & & & $\mathbf{X}$ \\
\hline & & & $\mathbf{X}$ \\
\hline & & & $\mathbf{X}$ \\
\hline & & & $\mathbf{X}$ \\
\hline & & & $\mathbf{X}$ \\
\hline & & & $\bar{X}$ \\
\hline & & $\mathbf{X}$ & $\mathbf{X}$ \\
\hline & & $\mathbf{X}$ & $\mathbf{X}$ \\
\hline & & $\mathbf{X}$ & $\mathbf{X}$ \\
\hline & & & $\mathbf{X}$ \\
\hline & & & $\mathbf{X}$ \\
\hline & & & $\bar{X}$ \\
\hline
\end{tabular}




\begin{tabular}{|l|l|l|l|}
\hline & & & $\mathbf{X}$ \\
\hline & & & $\mathbf{X}$ \\
\hline & & & $\mathbf{X}$ \\
\hline & & & $\mathbf{X}$ \\
\hline & & & $\mathbf{X}$ \\
\hline & & & $\mathbf{X}$ \\
\hline & & & $\mathbf{X}$ \\
\hline & & & $\mathbf{X}$ \\
\hline & $\mathbf{X}$ & & $\mathbf{X}$ \\
\hline & $\mathbf{X}$ & & $\mathbf{X}$ \\
\hline & $\mathbf{X}$ & & $\mathbf{X}$ \\
\hline & & & $\mathbf{X}$ \\
\hline & $\mathbf{X}$ & & $\mathbf{X}$ \\
\hline & $\mathbf{X}$ & & $\mathbf{X}$ \\
\hline & $\mathbf{X}$ & & \\
\hline
\end{tabular}




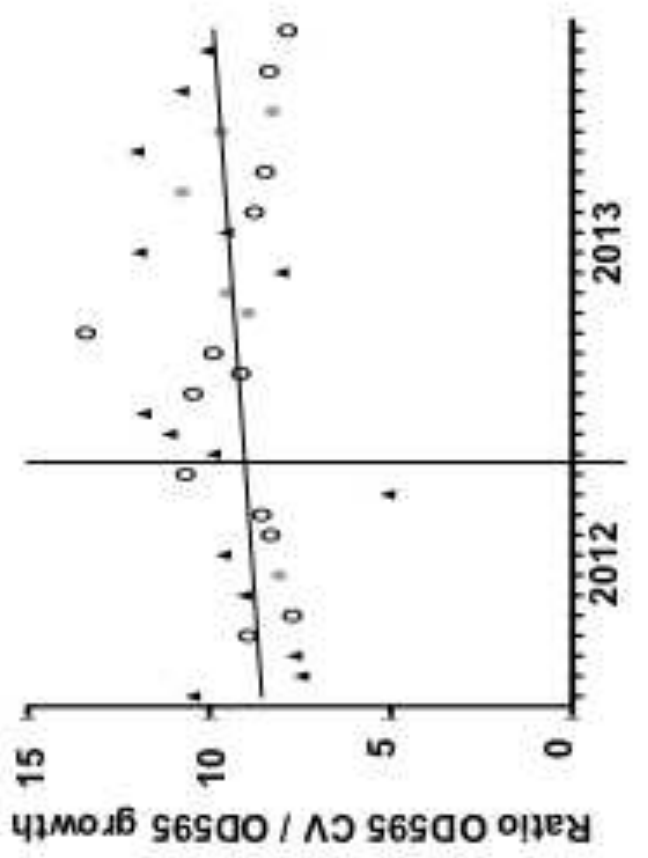

